\title{
S stars and s-process in the Gaia era
}

\section{Constraining the luminosity of the third dredge-up with Tc-rich S stars}

\author{
Shreeya Shetye ${ }^{1,2}$, Sophie Van Eck ${ }^{1}$, Alain Jorissen ${ }^{1}$, Stephane Goriely ${ }^{1}$, Lionel Siess ${ }^{1}$, Hans Van Winckel ${ }^{2}$, Bertrand \\ Plez $^{3}$, Michel Godefroid ${ }^{4}$, and George Wallerstein ${ }^{5}$
}
${ }^{1}$ Institute of Astronomy and Astrophysics (IAA), Université libre de Bruxelles (ULB), CP 226, Boulevard du Triomphe, B-1050 Bruxelles, Belgium e-mail: Shreeya. Shetye@ulb.ac.be
2 Institute of Astronomy, KU Leuven, Celestijnenlaan 200D, B-3001 Leuven, Belgium
3 Laboratoire Univers et Particules de Montpellier, Université de Montpellier, CNRS 34095, Montpellier Cedex 05, France
${ }^{4}$ Spectroscopy, Quantum Chemistry and Atmospheric Remote Sensing (SQUARES), CP160/09, Université libre de Bruxelles (ULB), 1050 Brussels, Belgium
5 Department of Astronomy, University of Washington, Box 351580, Seattle, WA 98195-1580, USA

Received; accepted

\section{ABSTRACT}

\begin{abstract}
Context. S stars are late-type giants that are transition objects between M-type stars and carbon stars on the asymptotic giant branch (AGB). They are classified into two types: intrinsic or extrinsic, based on the presence or absence of technetium (Tc). The Tc-rich or intrinsic S stars are thermally-pulsing (TP-)AGB stars internally producing s-process elements (including Tc) which are brought to their surface via the third dredge-up (TDU). Tc-poor or extrinsic S stars gained their s-process overabundances via accretion of s-process-rich material from an AGB companion which has since turned into a dim white dwarf.

Aims. Our goal is to investigate the evolutionary status of Tc-rich S stars by locating them in a Hertzsprung-Russell (HR) diagram using the results of Gaia early Data Release 3 (EDR3). We combine the current sample of 13 Tc-rich stars with our previous studies of 10 Tc-rich stars to determine the observational onset of the TDU in the metallicity range $[-0.7 ; 0]$. We also compare our abundance determinations with dedicated AGB nucleosynthesis predictions.

Methods. The stellar parameters are derived using an iterative tool which combines HERMES high-resolution spectra, accurate Gaia EDR3 parallaxes, stellar evolution models and tailored MARCS model atmospheres for S-type stars. Using these stellar parameters we determine the heavy-element abundances by line synthesis.

Results. In the HR diagram, the intrinsic $\mathrm{S}$ stars are located at higher luminosities than the predicted onset of the TDU. These findings are consistent with Tc-rich S stars being genuinely TP-AGB stars. The comparison of the derived s-process abundance profiles of our intrinsic S stars with the nucleosynthesis predictions provide an overall good agreement. Stars with highest [s/Fe] tend to have the highest $\mathrm{C} / \mathrm{O}$ ratios.
\end{abstract}

Key words. Stars: abundances - Stars: AGB and post-AGB - Hertzsprung-Russell and C-M diagrams - Nuclear reactions, nucleosynthesis, abundances - Stars: interiors

\section{Introduction}

S stars are late-type giants displaying, as the most characteristic feature of their optical spectra, ZrO bands (Merrill 1922) along with the usual $\mathrm{TiO}$ bands present in stars of similar temperatures (approximately 3000-4000 K, as M-type stars). The spectra show overabundances of s-process elements (Smith \& Lambert 1990) produced by the slow capture of neutrons on elements heavier than Fe (Burbidge et al. 1957; Käppeler et al. 2011) during the thermally-pulsing AGB phase (TP-AGB). These elements are then brought to the surface of the AGB star through mixing processes called third dredge-ups (TDU). The carbon over oxygen $(\mathrm{C} / \mathrm{O})$ ratio of $\mathrm{S}$ stars is in the range 0.5 to 0.99 which makes them transition objects between M-type $(\mathrm{C} / \mathrm{O} \sim 0.4)$ and carbon (C/O >1) stars (Iben \& Renzini 1983).

Another important characteristic of the S star family is the technetium (Tc) dichotomy. Tc is an element produced by the s-process and which has no stable isotope. Its isotope ${ }^{99} \mathrm{Tc}$ has a half-life of 210000 yrs. The puzzling detection of Tc in some
$S$ stars but not in others was solved after realizing that the $S$ stars without Tc (i.e. Tc-poor S stars) belong to binary systems. They are called extrinsic S stars (Smith \& Lambert 1988; Jorissen et al. (1993) because they owe their s-process abundances to mass transfer from a former AGB companion which is now a white dwarf. Therefore, the extrinsic S stars show s-process overabundances, except for Tc, which has decayed since the termination of the AGB phase of the companion. On the contrary, Tcrich $\mathrm{S}$ stars, called intrinsic, are producing s-process elements including Tc and transporting them to their surface via ongoing, recurrent TDU episodes.

We recently discovered a third class of $\mathrm{S}$ stars, the bitrinsic $\mathrm{S}$ stars (Shetye et al. 2020, S20 hereafter) that share properties with both the intrinsic and extrinsic classes: they are Tcrich and as such are located on the TP-AGB, but they also show overabundances of $\mathrm{Nb}$, a signature of extrinsically-enriched stars (Neyskens et al. 2015, Shetye et al. 2018; Karinkuzhi et al. 2018). Indeed, the unstable ${ }^{93} \mathrm{Zr}$ isotope (produced by the sprocess) decays into ${ }^{93} \mathrm{Nb}$, the only stable isotope of $\mathrm{Nb}$, in 
1.53 Myr. Therefore a niobium enrichment is expected in extrinsic stars, while it is not in TP-AGB stars.

The intrinsic S stars play an important role in our understanding of AGB nucleosynthesis and in particular of the TDU. They are the first objects on the AGB to show clear signatures ( $\mathrm{Tc}$ and $\mathrm{ZrO}$ ) of the TDU occurrences. They constrain the minimum luminosity of the TDU as well as its mass and metallicity dependence. The recent discovery of intrinsic, low-mass (initial mass $<1.5 \mathrm{M}_{\odot}$ ), solar metallicity S stars (Shetye et al. 2019, S19 hereafter) provided an observational evidence of the operation of TDUs even in low-mass stars.

In a previous paper (Shetye et al. 2018, S18 hereafter) we introduced a new method for the parameter and abundance determination in S-type stars. However, the number of intrinsic S stars available at the time of that study was limited by the bias of the Tycho-Gaia astrometric solution against extremely red sources (Michalik et al. 2015). In the current work we investigate the chemical composition and evolutionary status of 13 intrinsic $S$ stars from Gaia DR2 for which we could obtain high-resolution spectra. This paper is a follow up on the investigation of the evolutionary status of $\mathrm{S}$ stars conducted in the pioneering work of Smith \& Lambert (1990). We introduce the observational sample in Section 2 and discuss the Tc detection in Section 3 Sections 4 and 5 are dedicated to the description of the parameter and abundance determination. In Section 6 we present the Gaia EDR3 HR diagram of intrinsic and extrinsic S stars. We discuss the derived elemental abundances of the sample stars and compare them with nucleosynthesis predictions in Section 7 . Finally, we conclude with a summary of the most important results.

\section{Observational sample}

From the General Catalogue of Galactic S-Stars (Stephenson 1984, CGSS), we selected the ones with a Gaia Data Release 2 (DR2) parallax matching the condition $\sigma_{\varpi} / \varpi \leq 0.3$ and with available HERMES high-resolution spectra (Raskin et al.2011). From this sample we distinguished the intrinsic $\mathrm{S}$ stars from the extrinsic ones using Tc lines (Section 3) and kept only the Tc-rich S stars. Among these, the Tc-rich S stars with initial masses smaller than $1.5 \mathrm{M}_{\odot}$ were studied in S19. The cases of $\mathrm{BD}+79^{\circ} 156$ and $o^{1}$ Ori, two bitrinsic stars, were discussed in S20. In the current work we derive parameters and abundances for the remaining Tc-rich $S$ stars and also compile the Tc-rich S star results from S18 (3 stars), S19 (5 stars), S20 (2 stars) to increase the size of our sample.

Though the sample was designed using the Gaia DR2, Gaia early Data Release 3 (EDR3) became public during the course of our analysis. Hence the stellar parameters and luminosities were derived using Gaia EDR3. The relative parallax difference between the two releases $\left|\varpi_{\mathrm{DR} 2}-\varpi_{\mathrm{EDR} 3}\right| / \varpi_{\mathrm{EDR} 3}$ is usually smaller than $12 \%$ (except for V812 Oph, CSS 151, and CSS 454 which show the largest relative deviations of $22 \%, 33 \%$ and $57.5 \%$, respectively). The cause of the large parallax difference of some stars between DR2 and EDR3 is not yet clear. However, to make a consistent comparison of the present study with the previously studied samples (S18, S19 and S20), we re-computed the luminosities of all the extrinsic and intrinsic stars of S18, S19 and S20 using EDR3 (Table D.1). As a result, some stars previously identified (using DR2 parallaxes) as low-mass S stars (BD +34 1698 = CSS 413 and HD 357941 = CSS 1190; see S19) turn out to have higher masses $\left(\mathrm{M}_{\mathrm{ini}}=1.4\right.$ and $3.5 \mathrm{M}_{\odot}$ respectively $)$ according to their location in the HR diagram using EDR3 parallaxes (Sect. 6). This change in their masses required to adopt a $\log g$ value different from the one used for their abundance anal-
Table 1. The Tc-rich S stars excluded from the current study because of their large photometric variability.

\begin{tabular}{rcc}
\hline CGSS & AAVSO & $\Delta V(\mathrm{mag})$ \\
\hline 9 & $0018+38$ & 7.65 \\
307 & $0701+22$ & 7.53 \\
347 & $0720+46$ & 1.39 \\
856 & $1425+84$ & 2.95 \\
1219 & $2023+36$ & 1.10 \\
1115 & $1910-07$ & 4.76 \\
1309 & $2245+17$ & 4.74 \\
\hline
\end{tabular}

Notes. Columns 1 and 2 list the CGSS (Stephenson 1984) and AAVSO identifiers, respectively. Column 3 contains the $V$ amplitude from AAVSO.

ysis in S19 (both stars had $\log g \sim 1$ in S19; with EDR3 we find instead $\log g \sim 0$ for both of them). Hence, in the current work we also re-computed the stellar parameters and abundances of $\mathrm{BD}+34^{\circ} 1698$ and HD 357941, along the guidelines described in Sections. 4 and 5 using the EDR3 parallaxes (Table D.2).

A last criterion to define the Tc-rich S-star sample relates to the photometric variability. Large convective cells and/or pulsations are responsible for the photometric variability of TP-AGB stars. The thermal structure of these pulsating atmospheres can be significantly different from time-independent, hydrostatic atmospheres used to derive their stellar parameters, which can therefore be quite unreliable. We checked the time series data obtained by the American Association of Variable Star Observers (AAVSO) and removed from our sample the Tc-rich S stars with photometric variability $\Delta V>1 \mathrm{mag}$ (see Table 1) except for V812 Oph. The HERMES spectra of V812 Oph, despite its high variability $(\Delta V>1.4 \mathrm{mag})$, matched well the atmospheric models all over the optical range, hence we included it in the current sample. The sample of newly analyzed stars is listed in Table 2.

\section{Tc detection}

Three Tc I resonance lines located at 4238.19, 4262.27 and $4297.06 \AA$ were used. A signal to noise ratio $(\mathrm{S} / \mathrm{N})$ of at least 30 in the $V$-band is needed to detect the Tc lines in S-star spectra (Van Eck et al. 1998, S18, S19). The spectra of our sample stars all have a S/N (listed in Column 11 of Table 2) equal to or larger than 40 in the $V$ band and 30 in the $B$ band. The Tc absorption features of our sample stars around the three Tc lines are presented in Fig. 1 .

We confirm all previous Tc-rich classifications except for one target, HD 288833, classified as Tc-poor by Jorissen et al. (1993), despite the fact that its IRAS excess ([12] - [25]) is larger than -1.3 while extrinsic stars have [12] - [25] $<-1.3$ according to their classification criterion. This criterion was designed by Jorissen et al. (1993) to diagnose the expected lower infrared excess of the extrinsic stars with respect to that of (more evolved) intrinsic stars. The three Tc absorption features can be readily identified for this object in Fig. 1]

For all other stars our classification is in agreement with previous findings when they exist. HR Peg, AA Cam, V1139 Tau, V679 Oph and HD 64147 were indeed classified as intrinsic S stars in Smith \& Lambert (1988), Smith \& Lambert (1990) and Jorissen et al.(1993). We agree with Otto et al. [2011) classification of CSS 1152 as an intrinsic S star based on AKARI photometry and also confirm the results of Neyskens et al. (2015) that KR CMa and CSS 454 are intrinsic S stars. We could not use the Tc line at $4238.19 \AA$ for $\mathrm{CD}-27^{\circ} 5131$, as the region around 
Table 2. Basic data for our S star sample.

\begin{tabular}{rllccccccccc}
\hline CSS & Name & Sp. type & $V$ & $K$ & $J$ & $\begin{array}{c}\varpi_{\mathrm{EDR} 3} \pm \sigma_{\varpi} \\
(\mathrm{mas})\end{array}$ & $\begin{array}{c}Z_{5} \\
(\mathrm{mas})\end{array}$ & $E_{B-V}$ & $B C_{K}$ & Observation date & $\mathrm{S} / \mathrm{N}$ \\
\hline 89 & V1139 Tau & $\mathrm{S}$ & 7.90 & 0.97 & 2.27 & $1.79 \pm 0.05$ & -0.047 & 0.34 & 3.00 & 23 November 2012 & 150 \\
151 & CSS 151 & $\mathrm{S}$ & 10.99 & 4.64 & 5.97 & $0.36 \pm 0.03$ & -0.053 & 0.27 & 2.91 & 31 July 2017 & 40 \\
233 & HD 288833 & $\mathrm{S} 3 / 2$ & 9.40 & 4.26 & 5.51 & $0.83 \pm 0.02$ & -0.038 & 0.31 & 2.84 & 20 August 2012 & 100 \\
265 & KR CMa & M4S & 8.50 & 1.96 & 3.30 & $1.29 \pm 0.03$ & -0.030 & 0.11 & 3.01 & 10 April 2011 & 70 \\
312 & AA Cam & M5S & 7.79 & 1.39 & 2.59 & $2.08 \pm 0.05$ & -0.024 & 0.13 & 2.84 & 9 April 2011 & 90 \\
416 & HD 64147 & S5/2.5 & 9.20 & 3.61 & 5.00 & $0.56 \pm 0.03$ & -0.041 & 0.09 & 2.93 & 27 November 2016 & 40 \\
454 & CSS 454 & S & 10.39 & 4.93 & 6.13 & $0.40 \pm 0.03$ & -0.045 & 0.07 & 2.91 & 2 February 2017 & 50 \\
474 & CD -27 5131 & S4,2 & 9.62 & 2.72 & 4.35 & $0.64 \pm 0.03$ & -0.031 & 0.14 & 3.01 & 2 April 2010 & 70 \\
597 & BD -18 2608 & S & 9.58 & 4.01 & 5.27 & $0.71 \pm 0.02$ & -0.038 & 0.14 & 2.91 & 24 March 2018 & 70 \\
997 & V812 Oph & S5+/2.5 & 10.58 & 4.00 & 5.22 & $0.64 \pm 0.03$ & -0.053 & 0.22 & 2.93 & 22 April 2016 & 40 \\
1070 & V679 Oph & S5/5+ & 8.92 & 2.69 & 4.16 & $1.04 \pm 0.03$ & -0.049 & 0.37 & 2.83 & 16 March 2018 & 80 \\
1152 & Vy 12 & S5-/6 & 10.13 & 3.76 & 5.06 & $0.58 \pm 0.02$ & -0.037 & 0.21 & 2.99 & 18 March 2018 & 80 \\
1315 & HR Peg & S4+/1+ & 6.36 & 0.94 & 2.15 & $2.14 \pm 0.08$ & -0.043 & 0.12 & 2.93 & 17 July 2009 & 60 \\
\hline
\end{tabular}

Notes. Columns 1 and 2 list different identifiers: CGSS (Stephenson 1984) entry number and HD, BD or other names. Columns 3, 4, 5, and 6 indicate spectral type, $V, K$ and $J$ magnitudes, respectively, extracted from the SIMBAD Astronomical Database (Wenger et al. 2000). Column 7 lists the Gaia EDR3 parallax and its error. $Z_{5}$ is the five-parameter zero-point correction on the parallax from Lindegren et al. (2020). The reddenning $E_{B-V}$ has been obtained from the Gontcharov (2017) extinction maps. $B C_{K}$ is the bolometric correction in the $K$ band as computed from the MARCS model atmospheres. Columns 10 and 11 document the HERMES observation dates and their signal-to-noise (S/N) ratio around $500 \mathrm{~nm}$

this line is dominated by a cosmic ray hit. From its other two Tc lines we assess the Tc-rich nature of this object, in agreement with SVE17. For V812 Oph, CSS 151 and BD-18 ${ }^{\circ} 2608$, that we classify as Tc-rich, no literature classification is available.

\section{Derivation of the atmospheric parameters}

Disentangling the intricate parameter space of S stars has always been a challenging task because their spectra are dominated by molecules. The atmospheres of S stars are complex as the thermal structure is dependent on their chemical composition $(\mathrm{C} / \mathrm{O}$ and, to a lesser extent, heavy elements). Different methods have been used in the past for the stellar parameters determination. For instance, in their pioneering work, Smith \& Lambert (1985) derived $T_{\text {eff }}$ from the $(V-K)$ colors and $\log g$ from the standard $\log g$ - mass relationship where they estimated the masses by comparing the positions of the $S$ stars in the HR diagram with the evolutionary tracks. However, the $T_{\text {eff }}-(V-K)$ relation valid for M-type giants has been shown to be inappropriate for S-type stars (SVE17) because of the spectral energy distribution (SED) alteration (mainly by $\mathrm{ZrO}, \mathrm{LaO}$ and $\mathrm{YO}$ bands) induced by the non-standard chemical composition of S stars. New MARCS model atmospheres (Gustafsson et al. 2008) were designed to cover the full parameter range of S stars, with $T_{\text {eff }}$ from $2700 \mathrm{~K}$ to $4000 \mathrm{~K}, \log g$ from 0 to $5,[\mathrm{Fe} / \mathrm{H}]$ of 0.0 and $-0.5, \mathrm{C} / \mathrm{O}$ values of $0.500,0.752,0.899,0.925,0.951,0.971,0.991$ and [s/Fe] of 0.0, 1.0 and 2.0 (SVE17).

In the current study, the stellar parameters are determined as in S18. In summary, the high-resolution HERMES spectra are compared with a grid of $~ 3500$ synthetic spectra computed from the S star MARCS models to obtain atmospheric parameter estimates $T_{\text {eff }}, \log g$ and $[\mathrm{Fe} / \mathrm{H}]$. The fitting is undertaken over small spectral windows (listed in Appendix Table E.1 because HERMES spectra cannot be considered as flux-calibrated over their whole wavelength range. The fit with the lowest total $\chi^{2}$ value then identifies the best-fitting model, providing a first estimate of the atmospheric parameters.

The stellar luminosities were calculated using the distances derived from the Gaia EDR3 parallaxes after applying the zeropoint correction from Lindegren et al. (2020), the reddening $E_{B-V}$ computed from Gontcharov (2017) and the bolometric correction in the $K$ band as computed from the MARCS model atmospheres. Combining the $T_{\text {eff }}$, metallicity and luminosity, we located the stars in the HR diagram and compared them with STAREVOL (Siess 2006) evolutionary tracks to estimate their current stellar masses.

A new surface gravity was then computed and the procedure iterated (as described in Fig. 5 of S18) until the $\log g$ retrieved from spectral fitting was consistent with the one obtained from the HR diagram. The uncertainties on the stellar parameters were obtained from the variations of the atmospheric parameters while iterating for $\log g$. Our final set of parameters and the corresponding uncertainties are presented in Table 3 A further discussion of the reliability of the stellar masses thus found, based on their correlation with the height above the galactic plane and with 2MASS photometry, may be found in Appendix A

\section{Chemical abundance determination}

The abundance determination methodology is the same as the one adopted in S18 and S19. We compared the observed spectra with synthetic spectra generated using Turbospectrum v15.1 (Plez 2012) and MARCS model atmospheres of S stars with the parameters derived in Sect. 4. We have made use of the same 

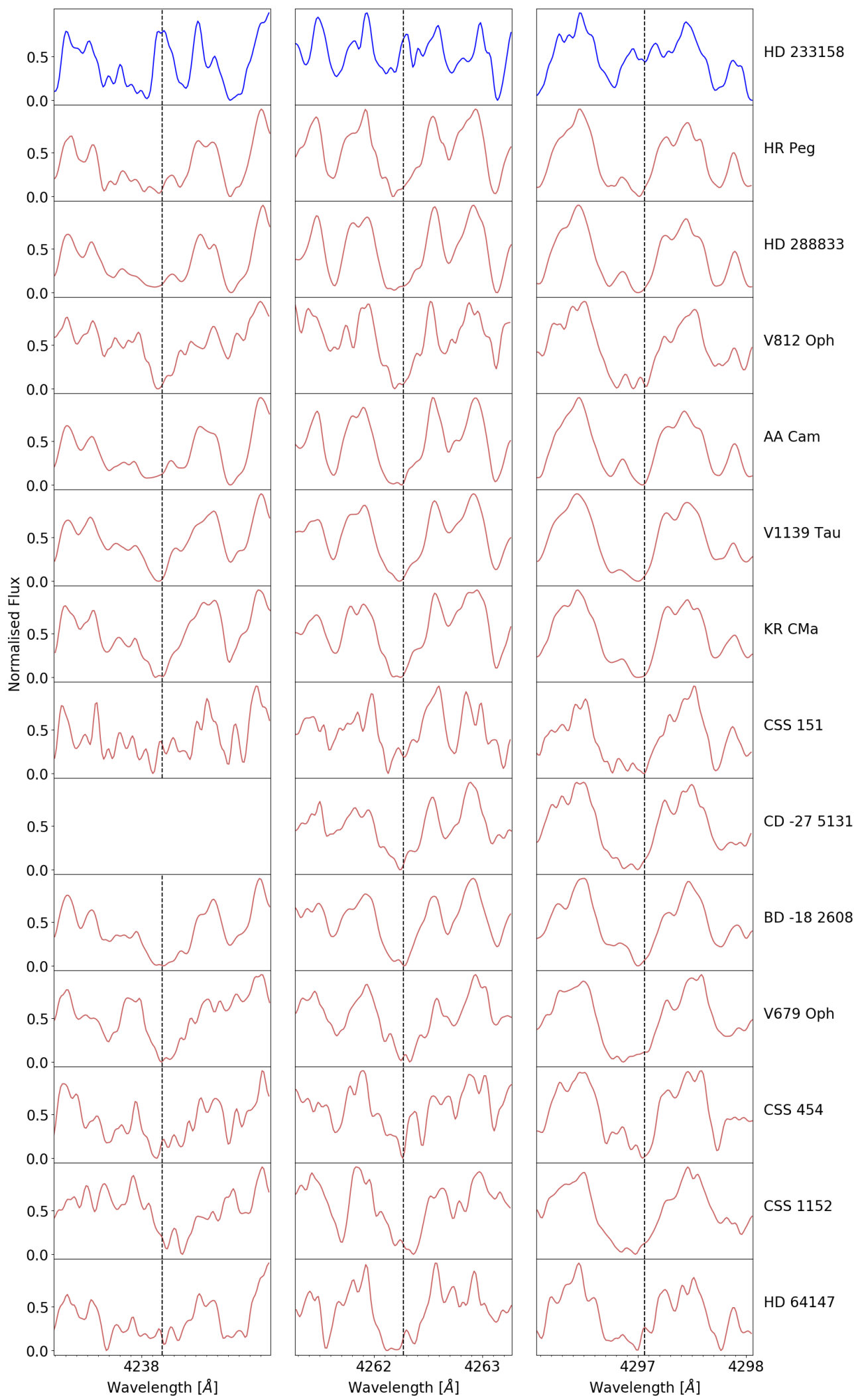

Fig. 1. The spectral region around the three $(4238.19,4262.27$ and $4297.06 \AA)$ Tc I lines in our sample of Tc-rich S stars. For comparison purposes, the spectrum of a Tc-poor S star (HD 233158, in blue in the top panels) from S18 is also plotted. The spectra have been arbitrarily normalized and binned by a factor of 1.5 to increase the $\mathrm{S} / \mathrm{N}$ ratio. The Tc I line at $4238.19 \AA$ A could not be used for CD $-27^{\circ} 5131$ as local normalization was hampered by a nearby cosmic ray hit.

input molecular line lists as in SVE17, atomic line list as in the
Gaia-ESO survey (Heiter et al.2020) and varied the abundances till a satisfactory agreement could be found. 
Table 3. Atmospheric parameters for $\mathrm{S}$ stars.

\begin{tabular}{|c|c|c|c|c|c|c|c|c|c|}
\hline Name & $\begin{array}{l}T_{\text {eff }} \\
(\mathrm{K})\end{array}$ & $\begin{array}{c}L \\
\left(L_{\odot}\right) \\
\end{array}$ & $\begin{array}{l}\log g \\
(\mathrm{dex})\end{array}$ & {$[\mathrm{Fe} / \mathrm{H}]$} & $\sigma_{[\mathrm{Fe} / \mathrm{H}]}$ & $\mathrm{C} / \mathrm{O}$ & $\begin{array}{c}{[\mathrm{s} / \mathrm{Fe}]} \\
(\mathrm{dex})\end{array}$ & $\begin{array}{l}\mathbf{M}_{\text {curr }} \\
\left(M_{\odot}\right) \\
\end{array}$ & $\begin{array}{l}\mathrm{M}_{\text {ini }} \\
\left(M_{\odot}\right) \\
\end{array}$ \\
\hline V1139 Tau & $\begin{array}{c}3400 \\
(3400-3500)\end{array}$ & $\begin{array}{c}6600 \\
(6200-6900)\end{array}$ & $\begin{array}{c}1 \\
(1-3)\end{array}$ & 3) & 0.13 & $\begin{array}{c}0.752 \\
(0.500-0.752)\end{array}$ & $\begin{array}{c}1 \\
(1-1)\end{array}$ & 3.3 & 3.5 \\
\hline CSS & $\begin{array}{c}3500 \\
(3500-3600)\end{array}$ & $\begin{array}{c}4600 \\
(4100-5200)\end{array}$ & $\begin{array}{c}1 \\
(1-3)\end{array}$ & $-0.25(14)$ & 0 & $\begin{array}{c}0.500 \\
(0.500-0.899)\end{array}$ & $\begin{array}{c}1 \\
(1-1)\end{array}$ & 2.6 & 3.2 \\
\hline HD 288 & $\begin{array}{c}3600 \\
(3600-3600)\end{array}$ & $\begin{array}{c}1700 \\
(1600-1800)\end{array}$ & $\begin{array}{c}1 \\
(1-2)\end{array}$ & $-0.30(13)$ & 0.14 & $\begin{array}{c}0.500 \\
(0.500-0.752)\end{array}$ & $\begin{array}{c}0 \\
(0-1)\end{array}$ & 1.3 & 1.4 \\
\hline KR CMa & $\begin{array}{c}3400 \\
400-3500)\end{array}$ & $\begin{array}{c}4800 \\
(4600-5000)\end{array}$ & $\begin{array}{c}1 \\
(1-3)\end{array}$ & -0. & 0 & $\begin{array}{c}0.500 \\
(0.500-0.752)\end{array}$ & $\begin{array}{c}1 \\
(1-1)\end{array}$ & 2.8 & 3.0 \\
\hline $\mathrm{AAC}$ & $\begin{array}{c}3600 \\
(3600-3600)\end{array}$ & $\begin{array}{c}3700 \\
(3600-3900)\end{array}$ & $\begin{array}{c}1 \\
(1-2)\end{array}$ & -0.3 & 0.13 & $\begin{array}{c}0.500 \\
(0.500-0.899)\end{array}$ & $\begin{array}{c}0 \\
(0-1)\end{array}$ & 2.4 & 2.5 \\
\hline $\mathrm{HD}$ & $\begin{array}{c}3500 \\
(3500-3600)\end{array}$ & $\begin{array}{c}5400 \\
(4900-5900)\end{array}$ & $\begin{array}{c}0 \\
(0-1)\end{array}$ & -0 . & 0 & $\begin{array}{c}0.500 \\
(0.500-0.752)\end{array}$ & $\begin{array}{c}0 \\
(0-1)\end{array}$ & 2.7 & 2.9 \\
\hline CSS & $\begin{array}{c}3500 \\
(3500-3600)\end{array}$ & $\begin{array}{c}2900 \\
(2600-3400)\end{array}$ & $\begin{array}{c}1 \\
(1-3)\end{array}$ & -0. & 0 & $\begin{array}{c}0.500 \\
(0.500-0.899)\end{array}$ & $\begin{array}{c}1 \\
(1-1)\end{array}$ & 1.8 & 1.9 \\
\hline $\mathrm{CD}-2$ & $\begin{array}{c}3400 \\
(3400-3600))\end{array}$ & $\begin{array}{c}9200 \\
(8300-10100)\end{array}$ & $\begin{array}{c}0 \\
(0-3)\end{array}$ & $-0.30(9)$ & 0.12 & $\begin{array}{c}0.500 \\
(0.500-0.899)\end{array}$ & $\begin{array}{c}1 \\
(1-1)\end{array}$ & 3.4 & 3.7 \\
\hline BD - & $\begin{array}{c}3500 \\
(3500-3600)\end{array}$ & $\begin{array}{c}2400 \\
(2300-2600)\end{array}$ & $\begin{array}{c}1 \\
(1-3)\end{array}$ & -0. & 0 & $\begin{array}{c}0.752 \\
(0.500-0.899)\end{array}$ & $\begin{array}{c}1 \\
(1-1)\end{array}$ & 1.5 & 1.6 \\
\hline V812 & $\begin{array}{c}3500 \\
(3500-3500)\end{array}$ & $\begin{array}{c}3000 \\
(2700-3200)\end{array}$ & $\begin{array}{c}1 \\
(1-2)\end{array}$ & $-0.37(13)$ & 0 & $\begin{array}{c}0.500 \\
(0.500-0.899)\end{array}$ & $\begin{array}{c}1 \\
(1-1)\end{array}$ & 1.8 & 2.0 \\
\hline V679 & $\begin{array}{c}3600 \\
(3600-3600)\end{array}$ & $\begin{array}{c}4600 \\
(4300-4800)\end{array}$ & $\begin{array}{c}1 \\
(1-1)\end{array}$ & $-0.52(9)$ & 0 . & $\begin{array}{c}0.899 \\
(0.752-0.899)\end{array}$ & $\begin{array}{c}1 \\
(1-2)\end{array}$ & 2.0 & 2.1 \\
\hline CSS 1 & $\begin{array}{c}3400 \\
(3400-3600)\end{array}$ & $\begin{array}{c}4400 \\
(4100-4700)\end{array}$ & $\begin{array}{c}1 \\
(1-2)\end{array}$ & $-0.14(6)$ & 0.13 & $\begin{array}{c}0.971 \\
(0.899-0.971)\end{array}$ & $\begin{array}{c}1 \\
(1-2)\end{array}$ & 2.8 & 3.0 \\
\hline HR Peg & $\begin{array}{c}3500 \\
(3500-3600)\end{array}$ & $\begin{array}{c}4900 \\
(4500-5200)\end{array}$ & $\begin{array}{c}0 \\
(0-4)\end{array}$ & $-0.34(13)$ & 0.17 & $\begin{array}{c}0.500 \\
(0.500-0.752)\end{array}$ & $\begin{array}{c}0 \\
(0-1)\end{array}$ & 2.2 & 2.3 \\
\hline
\end{tabular}

Notes. In the $T_{\text {eff }}, \log g$, and [s/Fe] columns, the numbers between brackets denote the values spanned during the log $g$ iterations, while in the $\mathrm{C} / \mathrm{O}$ column they indicate the error on $\mathrm{C} / \mathrm{O}$ from the spectral synthesis of the $\mathrm{CH}$ bands. In the $L$ column, they indicate the luminosity error due to the error on the Gaia EDR3 parallax. The numbers in brackets in the $[\mathrm{Fe} / \mathrm{H}]$ column indicate the number of Fe lines used to derive $[\mathrm{Fe} / \mathrm{H}]$ and the next column indicates the standard deviation (derived from the line-to-line scatter) on $[\mathrm{Fe} / \mathrm{H}]$. The current $\left(\mathrm{M}_{\text {curr }}\right)$ and initial $\left(\mathrm{M}_{\text {ini }}\right)$ masses have been derived from the locations of the stars in the HR diagram compared to STAREVOL tracks (Sect.6).

\section{1. $\mathrm{Li}$}

We used the $\mathrm{Li}$ I line at $6707.8 \AA$ to derive the $\mathrm{Li}$ abundance. This line is known to be blended with a neighboring Ce II line at $6708.099 \AA$ in the warmer post-AGB stars (Reyniers et al. 2002). However, we did not identify any dominating blend from this line to the $\mathrm{Li}$ I line in our sample stars. In Fig. 2 we present examples of stars with a good (bottom panel) as well as bad (top panel) spectral-synthesis fit of the considered Li line. We could derive the $\mathrm{Li}$ abundance for only three stars from our sample, namely HR Peg, V679 Oph and Vy 12. For these stars, the absorption features of the Li doublet located at $6707.8 \AA$ are very clear. For the rest of the sample, there were severe blends in the Li line (top panel of Fig. 2), so only upper limits on the Li abundances could be derived. The results are listed in Table C.1.

The Li abundances of all the intrinsic S stars of our sample are generally low except for HR Peg. HR Peg was found to be a Li-rich star also by Vanture et al. (2007) who classified the star as a high-mass star $\left(M>3 \mathrm{M}_{\odot}\right)$ with hot bottom burning (HBB) as an explanation for the $\mathrm{Li}$ abundance. However, our mass estimate for HR Peg is $M_{\text {curr }}=2.2 \mathrm{M}_{\odot}$, in agreement with the $2.0 \mathrm{M}_{\odot}$ value found by Neyskens et al. (2015). Therefore, HR Peg does not appear to be massive enough to be producing $\mathrm{Li}$ through HBB. Other plausible explanations involve some extra mixing in low-mass AGB stars (e.g. Charbonnel \& Balachandran
2000, Uttenthaler et al. 2007; Uttenthaler \& Lebzelter 2010) or the engulfment of planets/brown dwarfs (Siess \& Livio 1999).

\section{2. $C, N, O$}

We used the $\mathrm{CH}$ bands around $4250 \AA$ to determine the $\mathrm{C}$ abundance. The sensitivity of these bands to the carbon abundance is limited in S-star spectra because $\mathrm{CH}$ bands are blended with mainly $\mathrm{TiO}$ and $\mathrm{ZrO}$ (as can be seen from Fig. 16 of SVE17). It was not possible to derive the $\mathrm{O}$ abundance because the $\lambda 6300.3 \AA \mathrm{O}_{\mathrm{I}}$ line lies in a severely blended region. We used the solar oxygen abundance (Asplund et al. 2009) scaled with respect to the metallicity and included an alpha enhancement $([\alpha / \mathrm{Fe}]=-0.4 \times[\mathrm{Fe} / \mathrm{H}])$ for $[\mathrm{Fe} / \mathrm{H}] \geq-0.5$. The uncertainty on $\mathrm{C} / \mathrm{O}$ was estimated from the range of values of the $\mathrm{C} / \mathrm{O}$ providing an acceptable fit to the $\mathrm{CH}$ G-band.

The $\mathrm{N}$ abundance was then determined from the $\mathrm{CN}$ lines available in the region $8000-8100 \AA$. The line list for these CN lines was taken from Sneden et al. (2014).

\section{3. $[\mathrm{Fe} / \mathrm{H}]$}

We used the same line lists as S18 and S19 (see also Appendix Table F.1) for Fe line synthesis. Between 9 and $15 \mathrm{Fe}$ lines were used to derive the metallicity for all the sample stars, except for 

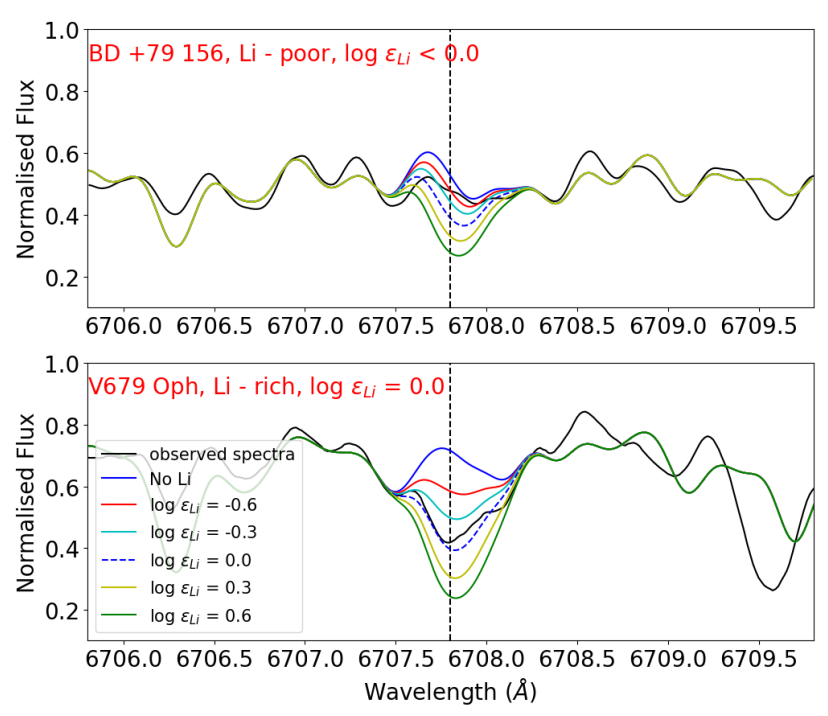

Fig. 2. Illustration of the fit quality between the observed and synthetic spectra of BD $+79^{\circ} 156$ (top panel) and V679 Oph (bottom panel) around the Li line at $6707.8 \AA$.

the star Vy 12, for which only six Fe lines could be used, as the other lines were strongly blended by molecules because of the high $\mathrm{C} / \mathrm{O}$ of this object (0.97). The derived metallicity as well as the standard deviation due to line-to-line scatter are listed in Table 3 .

\subsection{Light s-process elements ( $\mathrm{Sr}, \mathrm{Y}, \mathrm{Zr}, \mathrm{Nb})$}

The line list of S18 was used and complemented as documented in Appendix Table F.1 The strontium abundance could be derived only for HD 288833, BD-18 2608 , and V679 Oph. The Y abundance was measured for all the sample stars. The $\mathrm{Zr}$ abundance was measured for all stars except KR CMa using the two Zr I lines at 7819.37 and $7849.37 \AA$ with transition probabilities from laboratory measurements (Biémont et al. 1981). Some Nb I lines from Appendix Table F.1 could not be used in some stars. In fact, none of the $\mathrm{Nb}$ I lines could be used for CSS 151 because of the low $\mathrm{S} / \mathrm{N}$ of its spectrum. The $\mathrm{Nb}$ abundance could therefore not be derived for this star.

\subsection{Heavy s-process elements (Ba, $\mathrm{Ce}, \mathrm{Nd})$}

We derived the barium abundance using only one $\mathrm{Ba}$ I line located at $7488.07 \AA$. The cerium abundance was determined using the $\mathrm{Ce}$ II lines from S18 together with some new lines located at $7580.91,8025.57,8394.51,8405.25$, and $8769.91 \AA$. There is a huge scatter in the Ce abundances for HR Peg, $o^{1}$ Ori, KR CMa, $\mathrm{BD}+79^{\circ} 156$, and HD 64147 despite the use of Ce II lines displaying satisfactory fits. The bottom panel of Fig. 3illustrates the fact that even neighboring Ce II lines (at 8769.91 and $8772.13 \AA$ ) can provide discrepant abundances (by $\sim 0.4$ dex in this case). As explained in Karinkuzhi et al. (2018), cerium abundances derived using only Ce II lines above $7000 \AA$ are $\sim 0.3$ dex lower than the ones derived from Ce II lines in the range 4300-6500 $\AA$. These authors mention that this difference might be imputable to non-LTE (non-local thermodynamic equilibrium) effects. This could be the reason for the sub-solar cerium abundances in some of our stars, as they were determined using only red Ce II lines $(\lambda>7000 \AA)$. These uncertain abundances are marked with a colon in Tables C.2 and C.3

\subsection{Other heavy elements ( $P r, S m, E u)$}

Good Eu II and Pr II lines are located in the bluer part of the spectrum and could be used in some stars where the blending was weak. The $\mathrm{Sm}$ abundance was derived when possible using the Sm II 7042.20 and 7051.55 $\AA$ lines. These lines are well fitted and yield consistent abundances.

\section{7. $T c$}

All three Tc I resonance lines at 4238.19, 4262.27 and $4297.06 \AA$ are heavily blended (Little-Marenin \& Little 1979). We first derived the other s-process abundances in order to reproduce these blends as precisely as possible. The $4262.27 \AA$ line is the best reproduced by our spectral synthesis. Its blends, consisting in two neighbouring lines of $\mathrm{Nb}_{\mathrm{I}}$ (at $4262.050 \AA$ ) and Gd II (at $4262.087 \AA$ ) were identified in Van Eck \& Jorissen (1999). The Tc abundance was derived from the $4262.27 \AA$ Tc I line in our sample stars as well as in all intrinsic S stars from S18 and S19 (Table C.1).

\subsection{Uncertainties on the abundances}

The uncertainties on the abundances have been computed using the ones of the S star V915 Aql investigated in S18. The atmospheric parameters of V915 Aql are representative of those of most of our sample stars, hence we computed the elemental abundance error by quadratically adding the elemental standard deviation due to line-to-line scatter, the abundance errors due to parameter uncertainties of V915 Aql as derived in S18 (see also Appendix B], and a term of 0.1 dex to take into account continuum placement errors. For abundances that were derived using only one line, an arbitrary line-to-line scatter of $0.1 \mathrm{dex}$ was assumed. Error bars on Tc abundances were estimated as the range of Tc abundances providing an acceptable fit of the Tc I $4262.27 \AA$ line. The final elemental uncertainties on abundances are listed in Appendix Tables C.2, C.3 and C.4

\section{HR diagram of $\mathrm{S}$ stars}

In Fig. 4 we present an HR diagram collecting Tc-rich S stars studied in S18, S19, and S20 as well as those of the present work, together with Tc-poor stars from S18. We used the final stellar parameters presented in Table 2. The errors on $T_{\text {eff }}$ are taken from Table 2. For HD 288833, V812 Oph, AA Cam, and V679 Oph for which $T_{\text {eff }}$ did not change during the $\log g$ iterations, we imposed a standard symmetric error of $100 \mathrm{~K}$ on their $T_{\text {eff }}$. The asymmetric error on the luminosity was derived after propagating the error on the parallax. The evolutionary tracks displayed in Fig. 4 were computed with the STAREVOL code (Siess et al. 2000) and are described in detail in Escorza et al. (2017). Briefly, we use standard input physics with a mixing-length parameter $\alpha=1.75$, grey surface boundary conditions and the Asplund et al. (2009) solar mixture. Opacity enhancement due to the formation of molecules in carbon-rich atmospheres is also accounted for following the formulation of Marigo (2002). The Schröder \& Cuntz (2007) mass-loss prescription is activated up to the end of core helium burning followed by the Vassiliadis \& Wood (1993) formulation during the 

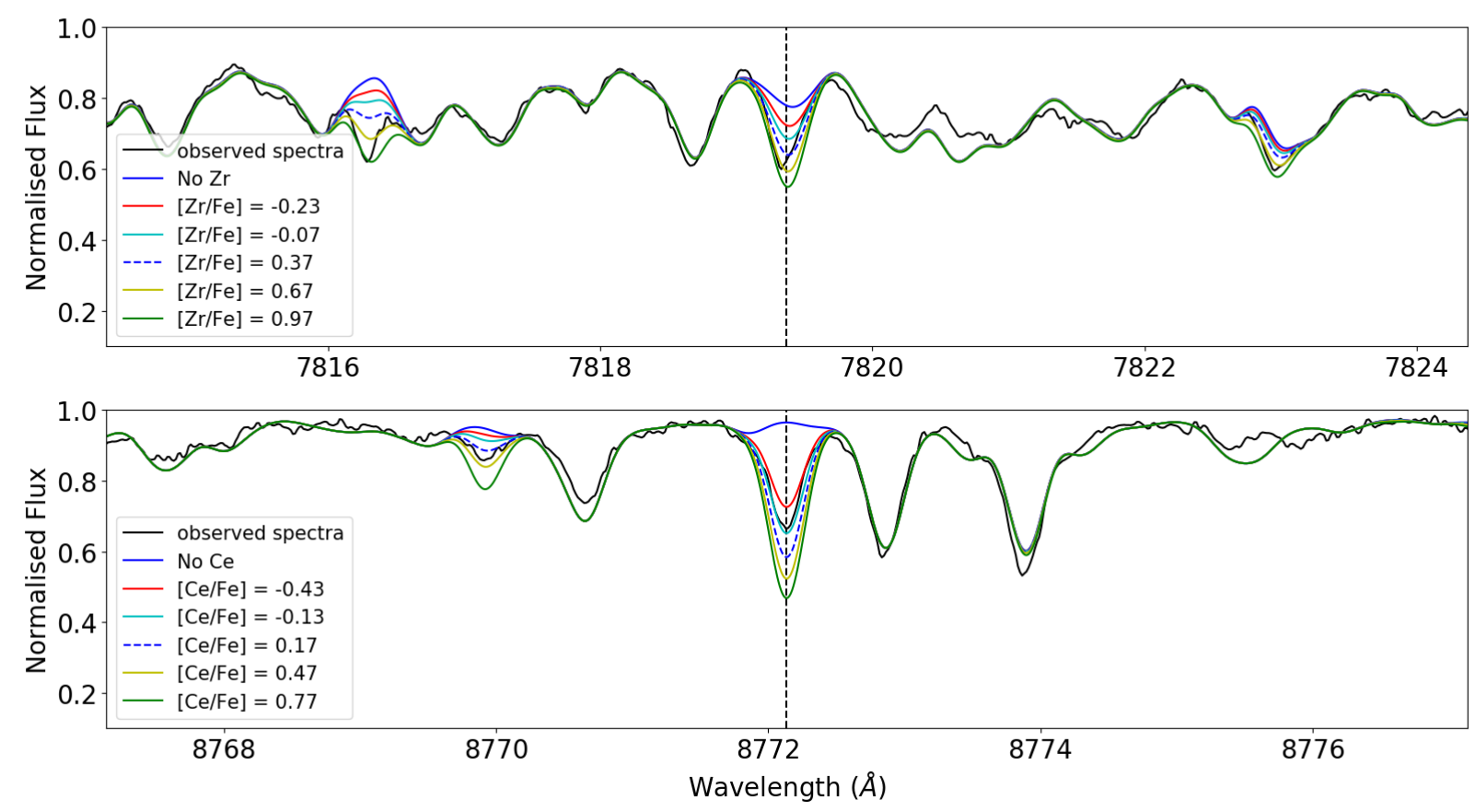

Fig. 3. Illustration of the fit between the observed and synthetic spectra for the S star HR Peg. The top panel presents $5 \AA$ around the Zr I line at $7819.37 \AA$ and the bottom panel around the Ce II line at $8772.135 \AA$.
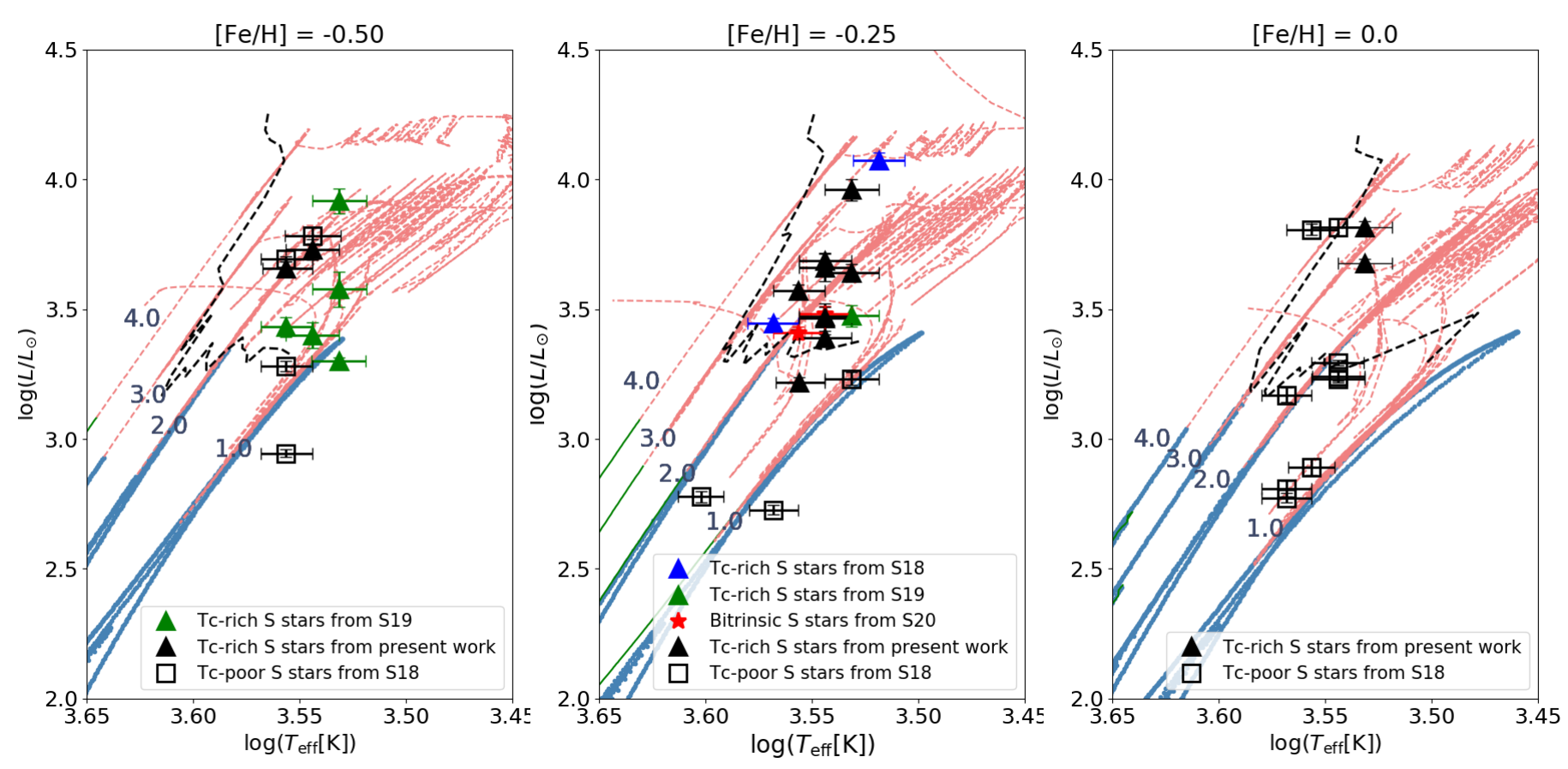

Fig. 4. HR diagram of intrinsic (filled triangles) $\mathrm{S}$ stars from our large sample and extrinsic (open squares) $\mathrm{S}$ stars from $\mathrm{S} 18$ along with the STAREVOL evolutionary tracks corresponding to the closest metallicities. The red giant branch is represented in blue, the core He-burning phase in green, whereas the red dashed lines correspond to the AGB tracks. The black dashed line represents the predicted onset of the third dredgeup, i.e., the lowest stellar luminosity following the first occurrence of a TDU episode (down to $1.5,1.3,1.0 \mathrm{M}_{\odot}$ for $[\mathrm{Fe} / \mathrm{H}]=0.0,-0.25,-0.50$ respectively).

AGB phase. We also consider overshooting below the convective envelope following the exponential decay expression of Herwig 
et al. (1997) with the parameter $f_{\text {over }}=0.01$. Models were computed for various metallicities, including $[\mathrm{Fe} / \mathrm{H}]=0,-0.25$ and -0.5 .

There is a clear segregation between the location of intrinsic and extrinsic S stars in the HR diagram. Indeed, whatever the considered metallicity range, intrinsic $\mathrm{S}$ stars are always located above the black dotted line (see Fig. 4) marking the predicted onset of TDU for masses above $1.5 \mathrm{M}_{\odot}$. Whereas it is obvious that Tc-rich stars must be TP-AGB stars (because of the Tc detection), such a consistency between the luminosity of the third dredge-up onset in theoretical models, and the luminosity of observed intrinsic S stars, which are the least evolved objects identified as TP-AGB stars, was not as clearly demonstrated so far.

Table 4 summarizes the observational constraints on the TDU first occurrence in terms of luminosity, initial mass and metallicity. For each mass and metallicity bins, we indicate the lowest luminosity of intrinsic S stars from our so-called large sample collecting stars from S18, S19, S20 and from the current work. These luminosities represent an observational upper limit on the TDU first occurrence in AGB stars, in the sense that Tcrich stars are observed at such luminosities, so that TDU must have already occurred by the time the star reaches this position in the HR diagram. However, since S stars are the first objects on the AGB to show clear signatures of TDU events (Tc and $\mathrm{ZrO}$ ), this upper limit must be quite close to the genuine TDU occurrence line. It can be used as an observational constraint to be satisfied by the stellar evolutionary models of the corresponding masses and metallicities.

\section{Discussion on the abundances and comparison with STAREVOL nucleosynthesis predictions}

The elemental abundances derived following the methodology presented in Sect. 5] allow us to investigate nucleosynthesis in intrinsic S stars. We compare the measured abundance profiles of our sample stars with the nucleosynthesis calculations of the STAREVOL code (Goriely \& Siess 2018) which uses an extended network of 411 species.

The predicted and measured abundance distributions are presented in Fig. 5. The pulse number is chosen in order to optimally match both the overabundance level and the location in the HR diagram. We find a good overall agreement between the predicted and measured distributions of heavy elements. In particular, the peak of heavy s-elements is well reproduced in all stars. However, problems persist with the light elements such as carbon or oxygen, as discussed in Sect. 7.1, and with some heavy elements, e.g. Ce as discussed in Sect. 5.4. The models do account for most of the derived Tc abundances; however, we note that abundance predictions for Tc are extremely sensitive to the pulse number and to the amount of dilution in the stellar envelope due to the initial absence of Tc in the star. Hence, the agreement between predicted and measured abundances may be poor in some cases, for e.g., CSS 151, BD -18²608, and CSS 454. We now investigate specific element ratios.

\section{1. $[\mathrm{C} / \mathrm{Fe}]$ and $[\mathrm{s} / \mathrm{Fe}]$}

The surface composition of TP-AGB stars should reflect the addition of ${ }^{12} \mathrm{C}$ originating from the He-burning shell and s-process material produced either radiatively in the interpulse or in the convective thermal pulses for more massive objects. The TDU is then responsible for transporting these products to the stellar surface. As the star evolves on the TP-AGB, its carbon abundance should thus increase along with its s-process over-abundances. Eventually the star becomes carbon-rich when the $\mathrm{C} / \mathrm{O}$ ratio exceeds unity. However, massive AGB stars with $M_{\text {ini }} \gtrsim 4 \mathrm{M}_{\odot}$ experience $\mathrm{HBB}$ and can efficiently burn the dredged-up carbon to produce mainly ${ }^{14} \mathrm{~N}$. In the top panel of Fig. 6 we present the carbon abundance as a function of s-process abundance for our intrinsic $\mathrm{S}$ star sample. The $[\mathrm{s} / \mathrm{Fe}]$ index has been calculated using the $\mathrm{Y}, \mathrm{Zr}$ and $\mathrm{Ba}$ abundances and is listed in Table C.5. In order to compare this trend with that of the stars from the next evolutionary stage, we added in Fig. 6 the Tc-rich carbon stars from Abia et al. (2002). A larger marker size indicates a lower metallicity (stars are grouped in three $[\mathrm{Fe} / \mathrm{H}]$ bins: $[-\infty,-0.4]$, $[-0.4,-0.2]$, and $[-0.2,0])$.

In the top panel of Fig. 6, the trend of increasing $[\mathrm{C} / \mathrm{Fe}]$ with increasing $[\mathrm{s} / \mathrm{Fe}]$, expected for TP-AGB stars, is not marked. Nevertheless, the $S$ stars most enriched in s-process elements are the ones with the highest carbon abundance. The carbon stars have higher carbon abundances than most S stars but are characterized by $[\mathrm{s} / \mathrm{Fe}]$ indices in the same range as those of the bulk of $\mathrm{S}$ stars. A group of intrinsic $\mathrm{S}$ stars have similar $[\mathrm{s} / \mathrm{Fe}]$ and $[\mathrm{C} / \mathrm{Fe}]$ ratios as carbon stars. This can be explained as follows: the carbon stars from Abia et al. (2002) have solar metallicities while the intrinsic $\mathrm{S}$ stars belonging to the group with similar $[\mathrm{C} / \mathrm{Fe}]$ (in the range 0.2 to $0.35 \mathrm{dex}$ ) have lower metallicities (in the range -0.2 to $-0.5 \mathrm{dex})$. Because $[\alpha / \mathrm{Fe}]$ increases with decreasing metallicity in the range $-0.5<[\mathrm{Fe} / \mathrm{H}]<0$, this group of $\mathrm{S}$ stars has a higher initial $[\mathrm{O} / \mathrm{Fe}]$ compared to carbon stars, enabling their O-rich classification.

The $[\mathrm{C} / \mathrm{Fe}]$ abundances in this work were derived from the $\mathrm{C} / \mathrm{O}$ ratios assuming solar oxygen abundance scaled with respect to metallicity and taking into account an $\alpha$-element enhancement (Sect. 5.2). The determination of $[\mathrm{C} / \mathrm{Fe}]$ is therefore quite indirect. We thus present in the bottom panel of Fig. 6 the $\mathrm{C} / \mathrm{O}$ ratios. Their determination is very robust, given the high sensitivity of molecular bands to the $\mathrm{C} / \mathrm{O}$ ratio. As expected, there is no overlap between $\mathrm{C} / \mathrm{O}$ ratio of $\mathrm{C}$ stars and S-type stars. We note that $3 \mathrm{~S}$-type stars have $\mathrm{C} / \mathrm{O}$ ratios close to, but definitely lower than 1 : indeed their $\mathrm{C} / \mathrm{O}$ of $0.899,0.971$, and 0.998 , produces an observed spectrum markedly different from the spectrum of an SC star (characterized by $\mathrm{C} / \mathrm{O}=1$ ).

A common misunderstanding is that $\mathrm{S}$ stars have $\mathrm{C} / \mathrm{O}=1$. This statement is often encountered in the literature even as a definition of S-type stars. Once again (see also Van Eck et al. 2017), we stress here that only $S$ stars with the highest [s/Fe] values have $\mathrm{C} / \mathrm{O}$ ratios approaching (but not reaching) unity. Most $\mathrm{S}$-type stars have intermediate $\mathrm{C} / \mathrm{O}$ ratios (between 0.5 and 0.8 ). Stars with $\mathrm{C} / \mathrm{O}=1$ are actually classified as SC or CS stars.

Figure 6 also compares the measured carbon and s-process abundances with the nucleosynthesis predictions at three different metallicities $([\mathrm{Fe} / \mathrm{H}]=0.0,-0.3$ and -0.5$)$. The filled circles along the tracks mark the thermal pulses turning the $\mathrm{C} / \mathrm{O}$ ratio to values above 1 , thus changing the (model) stars into carbon stars. First, the different models predict a tight correlation between $\mathrm{C} / \mathrm{O}$ and $[\mathrm{s} / \mathrm{Fe}]$, whereas there is a large scatter in measured $[\mathrm{s} / \mathrm{Fe}]$ at a given $\mathrm{C} / \mathrm{O}$. Second, theoretical calculations show a much faster increase of $\mathrm{C} / \mathrm{O}$ with $[\mathrm{s} / \mathrm{Fe}]$ than what is actually measured in stars. For example, all models predict that stars with $[\mathrm{s} / \mathrm{Fe}] \geq 0.55$ should have $\mathrm{C} / \mathrm{O}>1$ (and be carbon stars), whereas many $\mathrm{S}$ stars (which must have $\mathrm{C} / \mathrm{O}<1$ ) are observed with such large s-process enrichments. In other words, in theory, high s-process enrichments go along with very high $\mathrm{C} / \mathrm{O}$ ratios which are incompatible with $\mathrm{S}$-star classification. The reason why carbon stars do not show such large s-process over- 

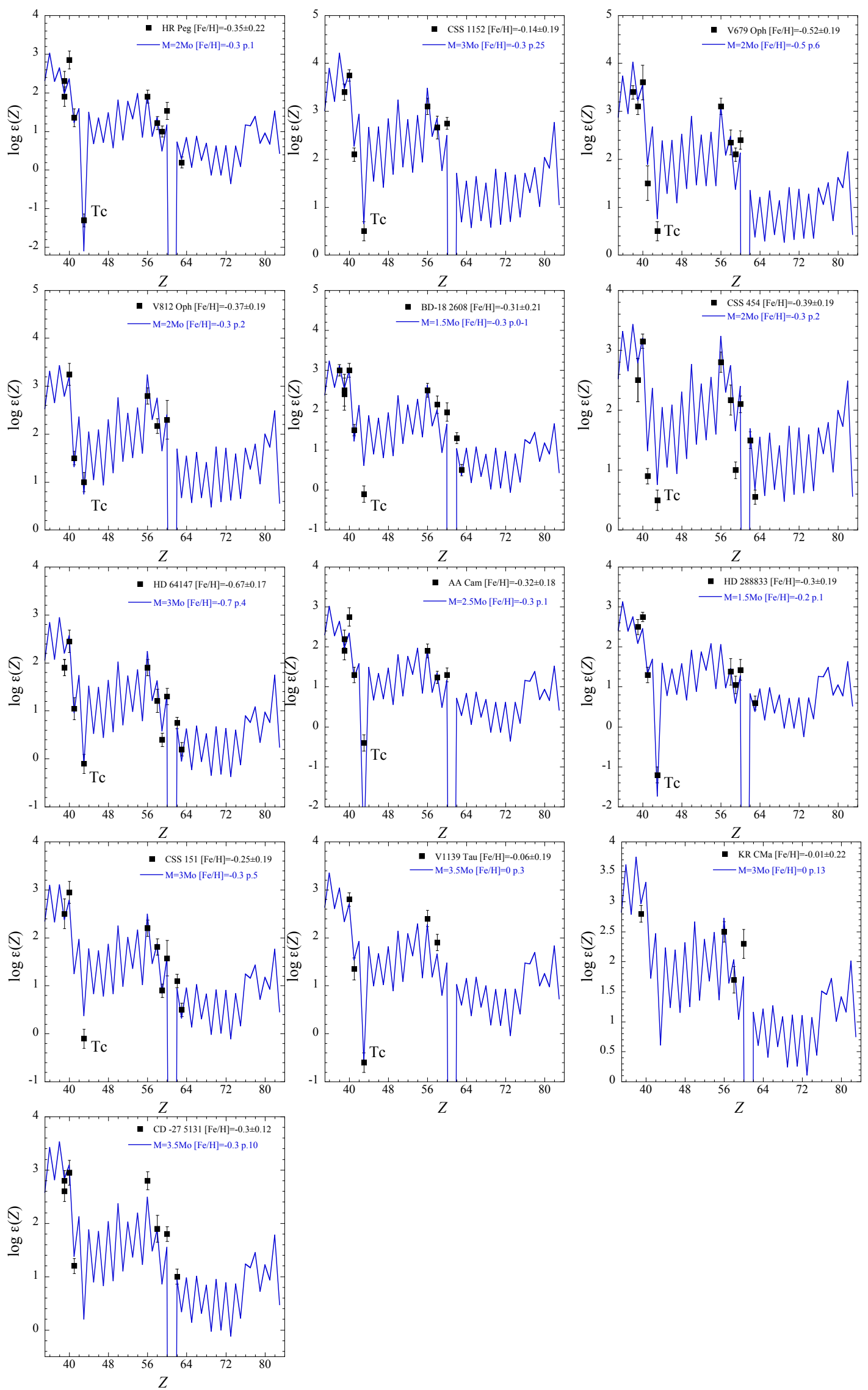

Fig. 5. Measured s-process element abundances compared with the nucleosynthesis predictions. The blue line represents the nucleosynthesis predictions compatible with the mass and metallicity of the individual stars. The number of pulses $n$ required to best-match the measured abundances is mentioned in the label of every panel as "p.n". 
Table 4. Observed lowest luminosities of intrinsic S stars in different mass and metallicity bins (collected from this work, S18, S19 and S20).

\begin{tabular}{c|l|c|c|c}
\hline \hline & Stars & Initial mass $\left(\mathrm{M}_{\odot}\right)$ & {$[\mathrm{Fe} / \mathrm{H}]$} & $L_{\mathrm{TDU}}\left(L_{\odot}\right)$ \\
\hline $\mathrm{M}_{\mathrm{ini}}<1.0 \mathrm{M}_{\odot}$ & V915 Aql & 1.0 & -0.5 & 2000 \\
\hline $1.0 \mathrm{M}_{\odot}<\mathrm{M}_{\mathrm{ini}}<1.5 \mathrm{M}_{\odot}$ & IRAS 06000+1023 (CSS 182) & 1.3 & -0.40 & 2500 \\
& HD 288833 & 1.4 & -0.30 & 1700 \\
\hline $1.5 \mathrm{M}_{\odot}<\mathrm{M}_{\text {ini }}<2.0 \mathrm{M}_{\odot}$ & BD -18 2608 & 1.6 & -0.31 & 2400 \\
& V812 Oph & 2.0 & -0.37 & 3000 \\
\hline $2.0 \mathrm{M}_{\odot}<\mathrm{M}_{\text {ini }}<2.5 \mathrm{M}_{\odot}$ & BD+79¹56 & 2.1 & -0.16 & 2600 \\
& V679 Oph & 2.1 & -0.52 & 4600 \\
\hline $2.5 \mathrm{M}_{\odot}<\mathrm{M}_{\text {ini }}<3.0 \mathrm{M}_{\odot}$ & HD 64147 & 2.9 & -0.67 & 5400 \\
& CSS 1152 & 3.0 & -0.14 & 4400 \\
& KR CMa & 3.0 & -0.01 & 4800 \\
\hline $\mathrm{M}_{\text {ini }}>3.0 \mathrm{M}_{\odot}$ & CSS 151 & 3.2 & -0.25 & 4600 \\
& V1139 Tau & 3.5 & -0.06 & 6600 \\
\hline \hline
\end{tabular}

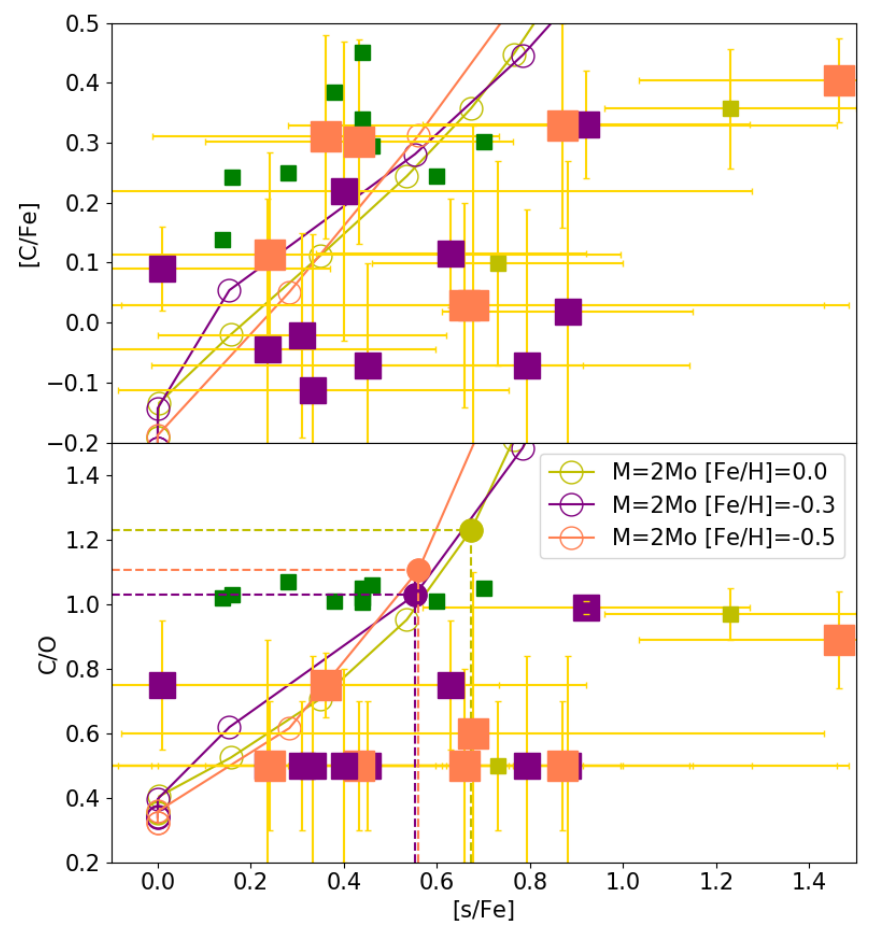

Fig. 6. Top panel: measured $[\mathrm{C} / \mathrm{Fe}]$ abundances as a function of $[\mathrm{s} / \mathrm{Fe}]$ for our large sample of Tc-rich S stars (light green, purple and orange squares) where the size of the symbol increases with decreasing metallicity, considering 3 metallicity bins: $[\mathrm{Fe} / \mathrm{H}]$ in $[0.0 ;-0.2]$ : light green, $[-0.2 ;-0.4]$ : purple, below -0.4 : orange. Tc-rich carbon stars from Abia et al. (2002) are shown as green squares. Bottom panel: derived $\mathrm{C} / \mathrm{O}$ ratio versus $[\mathrm{s} / \mathrm{Fe}]$ for the same stars. Predictions for a $2 \mathrm{M}_{\odot}$ STAREVOL model at metallicity $[\mathrm{Fe} / \mathrm{H}]=0.0$ (light green), -0.3 (violet) and -0.5 (orange) are overplotted. The empty circles along the tracks indicate the successive TDUs, while the three filled circles mark the first TDU allowing to reach $\mathrm{C} / \mathrm{O}>1$ in each model.

abundances (stronger line blending? dust obscuration of the most evolved objects?) is yet unclear.

Finally, we remark that Fig. 6 reveals the existence of one S star, $o^{1}$ Ori, with very mild - if any - enrichment in s-process elements (see $[\mathrm{s} / \mathrm{Fe}]$ in Table C.5. Figure 4 and Table A.1 from S20 for individual heavy-element abundances). This star is an- other example of the "Stephenson M-type stars" uncovered by Smith \& Lambert (1990). Nevertheless, it shows clear Tc signatures (Figure 1 of S20). The possible reasons for the low $[\mathrm{s} / \mathrm{Fe}]$ index of $o^{1}$ Ori are discussed in detail in S20 and Jorissen et al. (2019).
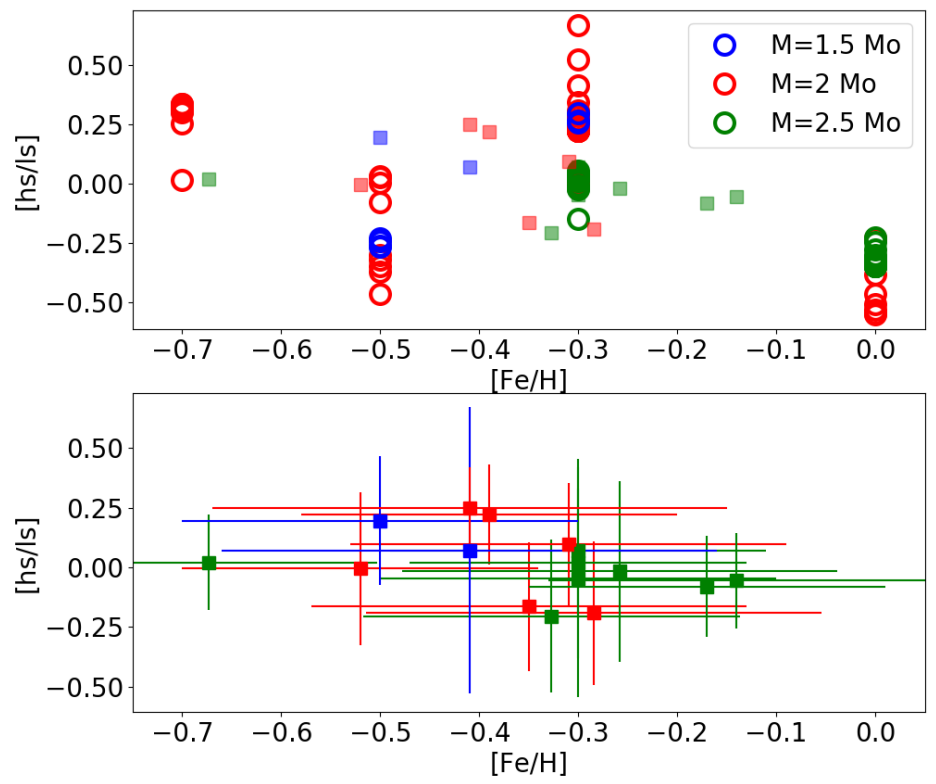

Fig. 7. The distribution of $[\mathrm{hs} / \mathrm{ls}]$ as a function of metallicity for the intrinsic $S$ stars of our study (filled squares). The hs-index has been calculated using $\mathrm{Ba}$ and $\mathrm{Nd}$, and the ls-index using $\mathrm{Y}$ and $\mathrm{Zr}$. The symbols are color-coded with respect to the initial mass in the bins $\mathbf{M}_{\text {ini }}<$ $1.5 \mathrm{M}_{\odot}$ (blue), $1.5 \leq \mathrm{M}_{\text {ini }}<2.5 \mathrm{M}_{\odot}$ (red), and $\mathrm{M}_{\text {ini }} \geq 2.5 \mathrm{M}_{\odot}$ (green). Note that these mass bins are designed to match the [hs/ls] STAREVOL predictions only available for $\mathrm{M}_{\mathrm{ini}}=1.5,2,2.5 \mathrm{M}_{\odot}$. Open circles denote the predictions from pulse to pulse for the different initial masses.

\section{2. [hs/ls] and metallicity}

We now investigate the potential correlations between the heavy (hs) to light (ls) s-process element ratio [hs/ls], mass and metallicity. The neutron irradiation index [hs/ls] is actually expected to increase with decreasing metallicity (Goriely \& Mowlavi 2000), as the number of neutrons per iron seed increases. 


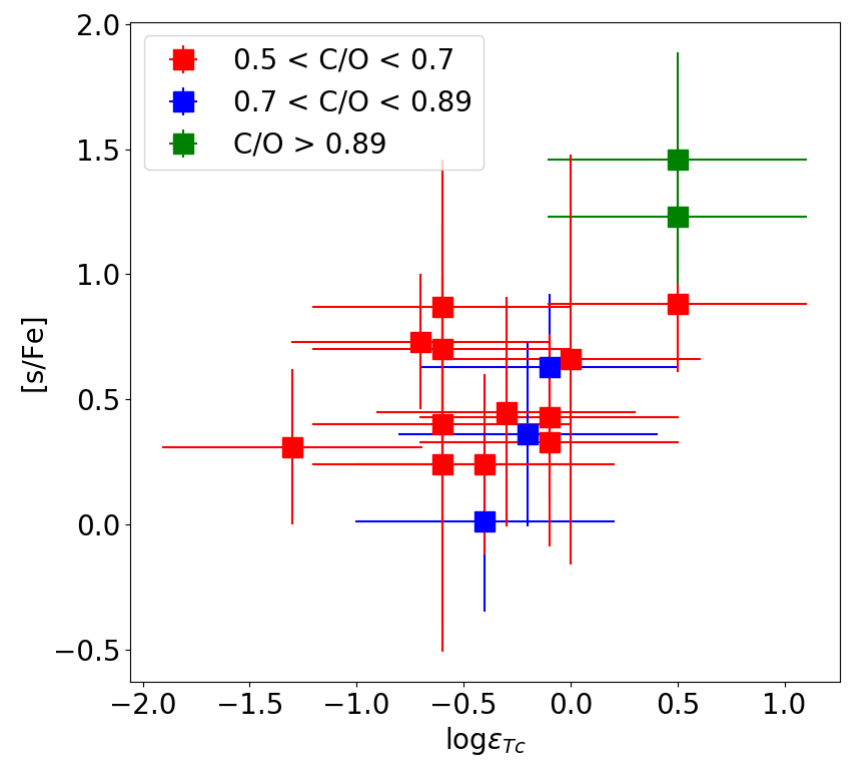

Fig. 8. $[\mathrm{s} / \mathrm{Fe}]$ index versus Tc abundance. The different colors represent different $\mathrm{C} / \mathrm{O}$ ratio bins as described in the figure.

From our measured abundances we find a large scatter in [hs/ls] with respect to metallicity (Fig. 7), within our limited range of metallicity. De Smedt et al. (2015, 2016) also reported such a scatter in the $[\mathrm{hs} / \mathrm{ls}]-[\mathrm{Fe} / \mathrm{H}]$ plane from their study of sprocess enriched post-AGB stars. However, the evolutionary link between post-AGB stars and intrinsic stars remains to be firmly established. Figure 7 also shows the absence of a clear correlation between the $[\mathrm{hs} / \mathrm{ls}]$ ratio and the initial stellar masses.

When compared with the theoretical predictions accounting for the pulse-to-pulse variation, plotted in the top panel of Fig. 7 . we find that the overall range covered by our measured [hs/ls] indices is compatible with that of the predicted abundances of the theoretical models. However, the [hs/ls] model predictions are not available for the complete metallicity range covered by our measured $[\mathrm{Fe} / \mathrm{H}]$.

\subsection{Technetium abundances}

\subsection{1. [s/Fe], $\mathrm{C} / \mathrm{O}$ and $\mathrm{Tc}$}

Figure 8 presents [s/Fe] (calculated as described in Sect. 7.1]using $\mathrm{Y}, \mathrm{Zr}$ and $\mathrm{Ba}$ abundances) as a function of the Tc abundance, for different $\mathrm{C} / \mathrm{O}$ ratio bins. The stars with the highest $[\mathrm{s} / \mathrm{Fe}]$ and Tc abundances are also the ones with the highest $\mathrm{C} / \mathrm{O}$ ratios, which is consistent with expectations from TP-AGB evolution. However, it is worth mentioning that whereas $[\mathrm{s} / \mathrm{Fe}]$ is predicted to monotonically increase as the star ascends its AGB (see Fig.6, this is also true for $\mathrm{C} / \mathrm{O}$ if there is no $\mathrm{HBB}$, and for luminosity, set aside the luminosity variations during thermal pulses), the technetium abundance has a more complex behaviour, because the ${ }^{99}$ Tc half life (210 $\left.000 \mathrm{yrs}\right)$ is not totally negligible with respect to the TP-AGB duration. For example, the evolution of the technetium-to-zirconium abundance ratio is not flat but shows a non-trivial evolution displayed in Fig. 2 of Neyskens et al. (2015). The lack of a clear trend in Fig. 8 is therefore not surprising.

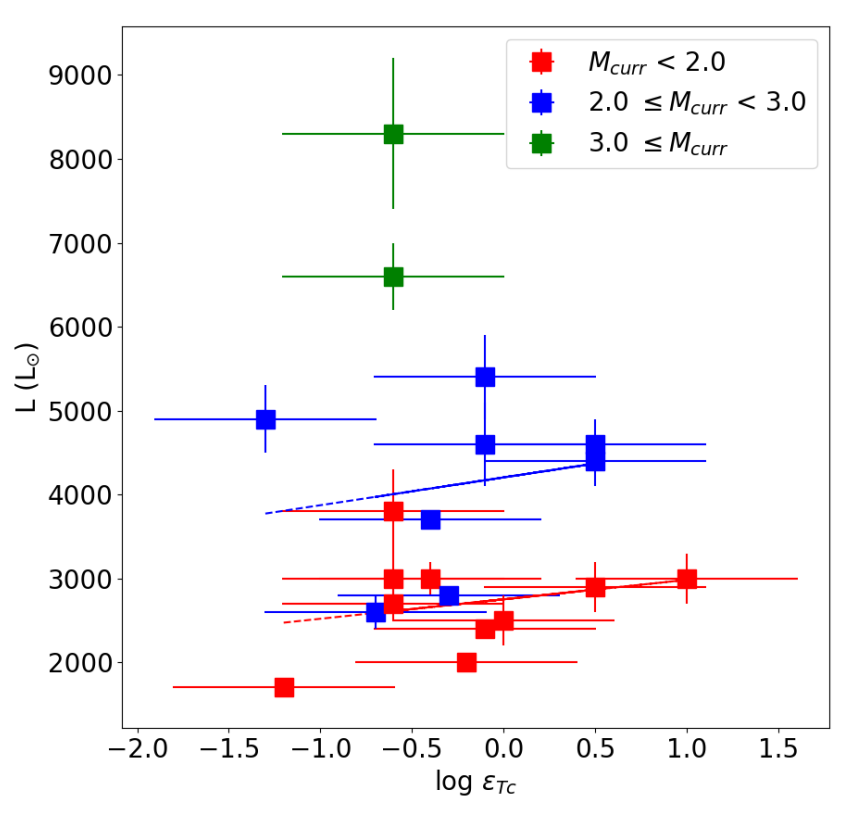

Fig. 9. Stellar luminosity as a function of Tc abundance. The symbols are color coded according to their mass bin. The blue and red dashed lines represent the linear least-squares fit for the stars in the corresponding mass bin.

\subsubsection{Luminosity and Tc}

In Fig. 9 we compare the Tc abundances and the luminosities of the sample stars. A large scatter in luminosity is present for each Tc abundance. If we focus on a specific mass bin, there is a loose trend of increasing Tc abundance with increasing luminosity, represented by the dashed least-square fit lines in Fig. 9 We also find that the most luminous and massive star in Fig. 9 has a relatively moderate Tc overabundance. A likely explanation is the larger dilution of s-elements in the bigger envelope of more massive AGB stars. Besides, with increasing stellar mass, the mass of the ${ }^{13} \mathrm{C}$ pocket responsible for the s-process, as well as that of the thermal pulses, are reduced because of the stronger compression of the shells induced by the larger core mass. Lower surface abundances of s-process elements are thus expected in higher-mass TP-AGB stars (García-Hernández et al. 2013).

\subsection{The Zr-Nb plane and intrinsic/extrinsic stars segregation}

A particular attention can also be paid to the $\mathrm{Zr}$ and $\mathrm{Nb}$ abundances that can be used as extrinsic/intrinsic star markers (Neyskens et al.2015). As already mentioned in Sect.1, niobium is mono-isotopic and can only be produced by the decay of ${ }^{93} \mathrm{Zr}$ (with a half-life of $1.53 \times 10^{6} \mathrm{yr}$ ). Intrinsic S stars are freshly producing s-process elements including ${ }^{93} \mathrm{Zr}$, and there is not enough time on the TP-AGB for a significant production of ${ }^{93} \mathrm{Nb}$. On the contrary, in extrinsic S stars, enough time has elapsed since the end of the nucleosynthesis in the companion for ${ }^{93} \mathrm{Zr}$ to have totally decayed into ${ }^{93} \mathrm{Nb}$. As expected, intrinsic and extrinsic $\mathrm{S}$ stars follow different trends in the $[\mathrm{Zr} / \mathrm{Fe}]-[\mathrm{Nb} / \mathrm{Fe}]$ plane, where intrinsic $S$ stars have $[\mathrm{Zr} / \mathrm{Nb}]<1$ while extrinsic stars have $[\mathrm{Zr} / \mathrm{Nb}] \sim 1$ (Neyskens et al. 2015, S18). The $\mathrm{Zr}-\mathrm{Nb}$ plane has been studied extensively for extrinsically-enriched objects: the high $\mathrm{Nb}$ abundance in extrinsic stars has been demon- 


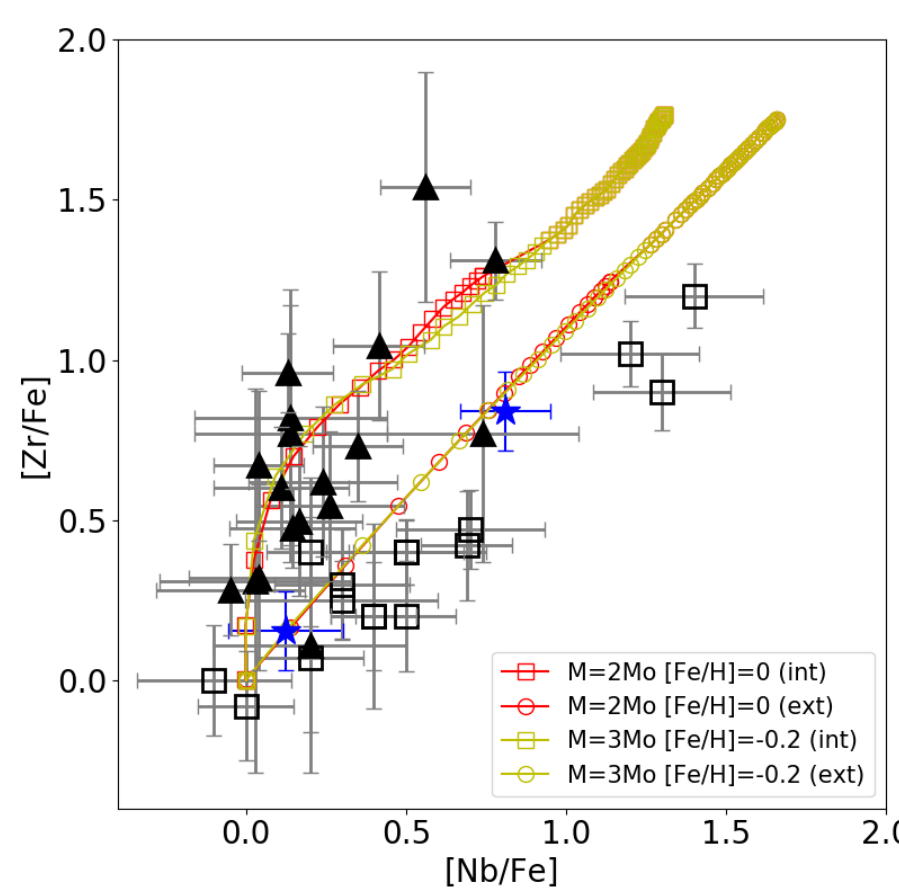

Fig. 10. $[\mathrm{Zr} / \mathrm{Fe}]$ as a function of $[\mathrm{Nb} / \mathrm{Fe}]$ for the intrinsic $\mathrm{S}$ stars (black filled triangles) and extrinsic S stars (open squares) from S18 compared with the nucleosynthesis predictions for different initial masses and metallicities. The bitrinsic S stars from S20 are plotted with a blue star symbol. The "ext" and "int" labels next to the model parameters refer to extrinsic (respectively intrinsic) model abundances.

strated in various extrinsic-star families among which $\mathrm{CH}$ and CEMP stars (Karinkuzhi et al. 2018, 2021). The apparent shift of extrinsic stars from the STAREVOL $2-3 \mathrm{M}_{\odot}$ predictions by a few tenths of a dex is as well discussed in Karinkuzhi et al. (2018). These authors speculated that this shift could arise due to the oscillator strengths of the two $\mathrm{Zr}$ lines used to derive the $\mathrm{Zr}$ abundance of the extrinsic S stars, which have a tendency to yield abundances that are about 0.1 dex too low in the benchmark stars of their sample (Arcturus and V762 Cas). Here we populate the high- $\mathrm{Zr}$, low- $\mathrm{Nb}$ region of this plane, adding constraints from the Tc-rich $\mathrm{S}$ stars.

Figure 10 confirms, on the basis of our so-called large sample of intrinsic $\mathrm{S}$ stars, the nice segregation between extrinsic and intrinsic stars in the $[\mathrm{Zr} / \mathrm{Fe}]-[\mathrm{Nb} / \mathrm{Fe}]$ plane (with one exception discussed below). We also compare the measured $\mathrm{Zr}$ and $\mathrm{Nb}$ abundances with nucleosynthesis predictions from STAREVOL for intrinsic and extrinsic stars of 2 and $3 \mathrm{M}_{\odot}$, and metallicities $[\mathrm{Fe} / \mathrm{H}]=0.0$ and -0.2 . In Fig. 10 , the intrinsic $\mathrm{S}$ stars from our sample have $[\mathrm{Zr} / \mathrm{Fe}]<1.6$ and follow the trend predicted for TP-AGB stars (open squares in red and green). It is worth mentioning that some ${ }^{93} \mathrm{Zr}$ decay (inducing some ${ }^{93} \mathrm{Nb}$ production) is both predicted and observed, as can be seen from the fact that highly-enriched (high-Zr) intrinsic stars, which are the most evolved S stars on the TP-AGB, tend to have the largest $\mathrm{Nb}$ enrichments, and nicely follow the STAREVOL inclined trend in the $[\mathrm{Zr} / \mathrm{Fe}]-[\mathrm{Nb} / \mathrm{Fe}]$ plane.

The two Tc-rich S stars $o^{1}$ Ori and BD+79 156 are 'bitrinsic' $S$ stars as they are Tc-rich (intrinsic) but show signs of binarity together with a large $\mathrm{Nb}$ enrichment (for the corresponding $[\mathrm{Zr} / \mathrm{Fe}]$ ratio), two evidences that they are also extrinsic stars, as discussed in S20. In addition to these two stars, the use of Gaia EDR3 parallaxes revealed a potentially new 'bitrinsic' candidate, $\mathrm{BD}+34^{\circ} 1698$. We re-computed the s-process abundances of this star as its stellar parameters (mainly mass and $\log g$ ) changed when Gaia DR2 parallaxes were replaced by Gaia EDR3. The revised s-process abundances of $\mathrm{BD}+34^{\circ} 1698$ are presented in Appendix Table D.2. It has a $[\mathrm{Zr} / \mathrm{Nb}]$ ratio close to unity, along with clear signatures of Tc (see Fig. C1 of S19), hence it qualifies as a 'bitrinsic' candidate. Wang \& Chen (2002) classified it as a candidate extrinsic $S$ star based on its IRAS photometry. Two spectra taken with the Hermes spectrograph revealed a clear radial-velocity variation: $V_{r}=18.59 \pm 0.05 \mathrm{~km} \mathrm{~s}^{-1}$ on JD 2457502.44 and $V_{r}=22.59 \pm 0.09 \mathrm{~km} \mathrm{~s}^{-1}$ on JD 2459289.51 . Our detection of the binary motion associated to a clear Tc enrichment and a $[\mathrm{Zr} / \mathrm{Nb}]$ ratio close to unity unambiguously classify $\mathrm{BD}+34^{\circ} 1698$ as a member of the restricted family of bitrinsic stars (S20). Lastly, Fig. 10 confirms that the $\mathrm{Zr}-\mathrm{Nb}$ analysis successfully serves as an additional test (apart from Tc) for the classification of S stars as intrinsic or extrinsic.

\section{Conclusions}

Thanks to the combination of Gaia EDR3 parallaxes and the 0 high-resolution HERMES spectra, we have determined the stellar parameters of a sample of 13 intrinsic $\mathrm{S}$ stars with metallicities in the range $-0.7<[\mathrm{Fe} / \mathrm{H}]<0$. We then derived their s-process element abundances. The heavy-element abundances of intrinsic S stars reveal their rich nucleosynthetic history. The main results from our study can be summarized as follows:

(i) The Gaia EDR3 HR diagram of S stars confirms that intrinsic $\mathrm{S}$ stars are more evolved than their extrinsic counterparts.

(ii) The luminosity lower limits for the occurrence of TDU in different mass and metallicity ranges provided in Table 4 can be used to constrain AGB evolutionary models.

(iii) The objects from our sample with the largest $\mathrm{C} / \mathrm{O}$ ratios are also the ones with the largest $[\mathrm{s} / \mathrm{Fe}]$, which is consistent with TP-AGB evolution predictions.

(iv) However, we clearly demonstrate the too rapid increase of the $\mathrm{C} / \mathrm{O}$ ratio with respect to $[\mathrm{s} / \mathrm{Fe}]$ in model predictions.

(v) The measured s-process abundances of intrinsic $\mathrm{S}$ stars are in good agreement with the AGB nucleosynthesis predictions for models of the corresponding mass and metallicity. In particular, the $\mathrm{Zr}$ and $\mathrm{Nb}$ abundances are matching very well the predicted trend for intrinsic S stars, confirming our previous finding (S18, $\mathrm{S} 20$ ) that the $\mathrm{Nb}$ abundance can be used as an intrinsic/extrinsic diagnostic as efficient as the Tc presence/absence.

(vi) We present Tc abundances for a large sample of intrinsic $\mathrm{S}$ stars (20 stars). We find that the stars with the highest $\mathrm{C} / \mathrm{O}$ ratios tend to be the ones with the highest Tc abundances.

In conclusion, the current investigation of a sample of intrinsic S stars has extended our understanding of their properties, in particular their location in the HR diagram and their chemical characterization considering $\sim 10$ chemical elements, including $\mathrm{C} / \mathrm{O}$ ratio and the radioactive element technetium.

Acknowledgements. The authors thank the anonymous referee for constructive comments. This research has been funded by the Belgian Science Policy Office under contract BR/143/A2/STARLAB. SS, SVE, SG, MG acknowledge support from the FWO \& FNRS Excellence of Science Programme (EOS-O022818F). SVE thanks Fondation ULB for its support. GW's research is supported by The Kennilworth Fund of the New York Community Trust. Based on observations obtained with the HERMES spectrograph, which is supported by the Research Foundation - Flanders (FWO), Belgium, the Research Council of KU Leuven, Belgium, the Fonds National de la Recherche Scientifique (F.R.S.-FNRS), Belgium, the Royal Observatory of Belgium, the Observatoire de Genève, Switzer- 
land and the Thüringer Landessternwarte Tautenburg, Germany. This work has made use of data from the European Space Agency (ESA) mission Gaia (https://www.cosmos.esa.int/gaia) processed by the Gaia Data Processing and Analysis Consortium (DPAC, https://www. cosmos.esa.int/ web/gaia/dpac/consortium) Funding for the DPAC has been provided by national institutions, in particular the institutions participating in the Gaia Multilateral Agreement. This research has also made use of the SIMBAD database, operated at CDS, Strasbourg, France. LS \& SG are senior FNRS research associates.

\section{References}

Abia, C., Domínguez, I., Gallino, R., et al. 2002, ApJ, 579, 817

Asplund, M., Grevesse, N., Sauval, A. J., \& Scott, P. 2009, ARA\&A, 47, 481

Biémont, E., Grevesse, N., Hannaford, P., \& Lowe, R. M. 1981, ApJ, 248, 867

Burbidge, E. M., Burbidge, G. R., Fowler, W. A., \& Hoyle, F. 1957, Reviews of Modern Physics, 29, 547

Charbonnel, C. \& Balachandran, S. C. 2000, A\&A, 359, 563

Corliss, C. H. \& Bozman, W. R. 1962, NBS Monograph, Vol. 53, Experimental transition probabilities for spectral lines of seventy elements; derived from the NBS Tables of spectral-line intensities (US Government Printing Office)

De Smedt, K., Van Winckel, H., Kamath, D., et al. 2016, A\&A, 587, A6

De Smedt, K., Van Winckel, H., Kamath, D., \& Wood, P. R. 2015, A\&A, 583, A56

Den Hartog, E. A., Lawler, J. E., Sneden, C., \& Cowan, J. J. 2003, ApJS, 148, 543

Duquette, D. W. \& Lawler, J. E. 1982, Phys. Rev. A, 26, 330

Escorza, A., Boffin, H. M. J., Jorissen, A., et al. 2017, A\&A, 608, A100

García, G. \& Campos, J. 1988, J. Quant. Spec. Radiat. Transf., 39, 477

García-Hernández, D. A., Zamora, O., Yagüe, A., et al. 2013, A\&A, 555, L3

Gontcharov, G. A. 2017, Astronomy Letters, 43, 472

Goriely, S. \& Mowlavi, N. 2000, A\&A, 362, 599

Goriely, S. \& Siess, L. 2018, A\&A, 609, A29

Gustafsson, B., Edvardsson, B., Eriksson, K., et al. 2008, A\&A, 486, 951

Heiter, U., Lind, K., Bergemann, M., et al. 2020, arXiv e-prints, arXiv:2011.02049

Herwig, F., Bloecker, T., Schoenberner, D., \& El Eid, M. 1997, A\&A, 324, L81

Iben, Jr., I. \& Renzini, A. 1983, ARA\&A, 21, 271

Jorissen, A., Boffin, H. M. J., Karinkuzhi, D., et al. 2019, A\&A, 626, A127

Jorissen, A., Frayer, D. T., Johnson, H. R., Mayor, M., \& Smith, V. V. 1993, A\&A, 271, 463

Käppeler, F., Gallino, R., Bisterzo, S., \& Aoki, W. 2011, Reviews of Modern Physics, 83, 157

Karinkuzhi, D., Van Eck, S., Goriely, S., et al. 2021, A\&A, 645, A61

Karinkuzhi, D., Van Eck, S., Jorissen, A., et al. 2018, A\&A, 618, A32

Kupka, F., Piskunov, N., Ryabchikova, T. A., Stempels, H. C., \& Weiss, W. W. 1999, A\&AS, 138, 119

Kurucz, R. L. 2007, Robert L. Kurucz on-line database of observed and predicted atomic transitions

Lebzelter, T., Mowlavi, N., Marigo, P., et al. 2018, A\&A, 616, L13

Lindegren, L., Bastian, U., Biermann, M., et al. 2020, arXiv e-prints, arXiv:2012.01742

Little-Marenin, I. R. \& Little, S. J. 1979, AJ, 84, 1374

Marigo, P. 2002, A\&A, 387, 507

Martin, G., Fuhr, J., \& Wiese, W. 1988, J. Phys. Chem. Ref. Data Suppl., 17

Meggers, W. F., Corliss, C. H., \& Scribner, B. F. 1975, Tables of spectral-line intensities. Part I, II - arranged by elements (US Government Printing Office)

Merrill, P. W. 1922, ApJ, 56, 457

Michalik, D., Lindegren, L., \& Hobbs, D. 2015, A\&A, 574, A115

Miles, B. M. \& Wiese, W. L. 1969, Atomic Data, 1, 1

Nave, G., Johansson, S., Learner, R. C. M., Thorne, A. P., \& Brault, J. W. 1994, ApJS, 94, 221

Neyskens, P., Van Eck, S., Jorissen, A., et al. 2015, Nature, 517, 174

Nilsson, A. E., Johansson, S., \& Kurucz, R. L. 1991, Phys. Scr, 44, 226

O’Brian, T. R., Wickliffe, M. E., Lawler, J. E., Whaling, W., \& Brault, J. W. 1991, Journal of the Optical Society of America B Optical Physics, 8, 1185

Otto, E., Green, P. J., \& Gray, R. O. 2011, ApJS, 196, 5

Palmeri, P., Fischer, C. F., Wyart, J. F., \& Godefroid, M. R. 2005, MNRAS, 363, 452

Palmeri, P., Quinet, P., Wyart, J., \& Biémont, E. 2000, Physica Scripta, 61, 323

Plez, B. 2012, Turbospectrum: Code for spectral synthesis, Astrophysics Source Code Library

Raskin, G., van Winckel, H., Hensberge, H., et al. 2011, A\&A, 526, A69

Reyniers, M., Winckel, H., Biemont, E., \& Quinet, P. 2002, Astronomy and Astrophysics, 395

Schröder, K.-P. \& Cuntz, M. 2007, A\&A, 465, 593

Shetye, S., Goriely, S., Siess, L., et al. 2019, A\&A, 625, L1

Shetye, S., Van Eck, S., Goriely, S., et al. 2020, A\&A, 635, L6
Shetye, S., Van Eck, S., Jorissen, A., et al. 2018, A\&A, 620, A148

Siess, L. 2006, A\&A, 448, 717

Siess, L., Dufour, E., \& Forestini, M. 2000, A\&A, 358, 593

Siess, L. \& Livio, M. 1999, MNRAS, 304, 925

Smith, V. V. \& Lambert, D. L. 1985, ApJ, 294, 326

Smith, V. V. \& Lambert, D. L. 1988, ApJ, 333, 219

Smith, V. V. \& Lambert, D. L. 1990, ApJS, 72, 387

Sneden, C., Lucatello, S., Ram, R. S., Brooke, J. S. A., \& Bernath, P. 2014, ApJS, 214, 26

Stephenson, C. B. 1984, Publications of the Warner \& Swasey Observatory, 3, 1 Uttenthaler, S. \& Lebzelter, T. 2010, A\&A, 510, A62

Uttenthaler, S., Lebzelter, T., Palmerini, S., et al. 2007, A\&A, 471, L41

Van Eck, S. \& Jorissen, A. 1999, A\&A, 345, 127

Van Eck, S., Jorissen, A., Udry, S., Mayor, M., \& Pernier, B. 1998, A\&A, 971

Van Eck, S., Neyskens, P., Jorissen, A., et al. 2017, A\&A, 601, A10

Vanture, A. D., Smith, V. V., Lutz, J., et al. 2007, PASP, 119, 147

Vassiliadis, E. \& Wood, P. R. 1993, ApJ, 413, 641

Wang, X. H. \& Chen, P. S. 2002, A\&A, 387, 129

Wenger, M., Ochsenbein, F., Egret, D., et al. 2000, A\&AS, 143, 9

Xu, H. L., Svanberg, S., Quinet, P., Garnir, H. P., \& Biémont, E. 2003, Journal of Physics B Atomic Molecular Physics, 36, 4773

\section{Appendix A: Reliability of the S-star masses}

\section{Appendix A.1: Height above the galactic plane}

Figure A.1 presents the height above the galactic plane $(|Z|)$ of our sample stars. Though a large $Z$ scatter is present at low masses, the maximum $|Z|$ value decreases with increasing mass, as expected. This trend somehow validates the masses estimated in the present paper. Nevertheless, the limited size of the present sample, and the absence of bias control in this sample, precludes us from drawing any further conclusion.

\section{Appendix A.2: Gaia-2MASS photometry}

Lebzelter et al. (2018) present a classification of AGB stars using Gaia and 2MASS photometry, in the plane $W_{R P, B P-R P}-W_{K, J-K}$ vs $M_{K}$, where $M_{K}$ is the absolute $K_{S}$ magnitude from 2MASS and Gaia EDR3 parallaxes. The Wesenheit functions $W_{R P, B P-R P}$ and $W_{K, J-K}$ are calculated using the definitions from Lebzelter et al. (2018), i.e., $W_{K, J-K}=K_{S}-0.686(J-K)$ and $W_{R P}=G_{R P}-1.3\left(G_{B P}-G_{R P}\right)$, with $J$ and $K_{S}$ from 2MASS, and $G_{B P}$ and $G_{R P}$ are the magnitudes in the Gaia $B P$ and $R P$ bands. In Fig. A.2, we plot our sample stars in this classification scheme. Most of our sample stars with masses smaller than $2.5 \mathrm{M}_{\odot}$ lie as expected in the "oxygen-rich low-mass AGB" zone. While an oxygen-rich chemistry is indeed expected for S-type stars (which have $\mathrm{C} / \mathrm{O}$ smaller than unity), their location in the low-mass zone constitutes a nice confirmation of the masses derived in the present work. The case of HD 357941, which was flagged as a low-mass star $\left(M_{\text {ini }} \sim 1 \mathrm{M}_{\odot}\right)$ based on DR2 parallaxes in $\mathrm{S} 19$, is noteworthy. We re-evaluated its mass with Gaia EDR3 parallaxes and found it to be $M_{\text {ini }}=3.5 \mathrm{M}_{\odot}$. This revised mass is now consistent with its location in Fig. A.2 $\left(M_{K}=-7.94, W_{R P, B P-R P}-W_{K, J-K}=-0.03\right)$, where it is located at the border between the low-mass and intermediate-mass $\mathrm{O}$ rich AGB stars.

\section{Appendix B: Error analysis of the Tc and $\mathrm{Li}$ abundances}

Uncertainties on the $\mathrm{Li}$ and $\mathrm{Tc}$ abundances of V915 Aql are listed in the bottom panel of Table B.1. In the upper panel, model A designates the adopted model for V915 Aql from S18, whereas models B-G correspond to models differing by one grid step from model A, each parameter varied at a time. The abundances resulting from each of these models is then compared 

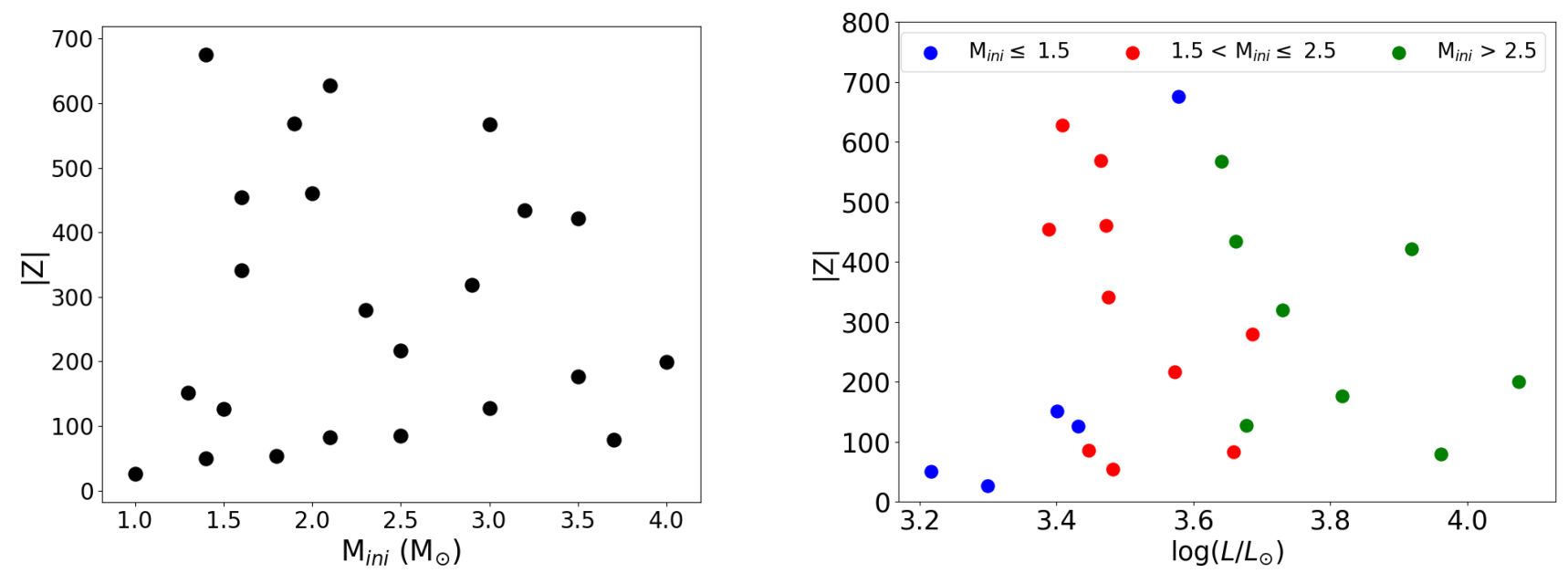

Fig. A.1. Height above the galactic plane for the sample intrinsic stars and for the intrinsic stars from S18, S19 and S20, as a function of their initial mass (left panel) or luminosity (right panel).

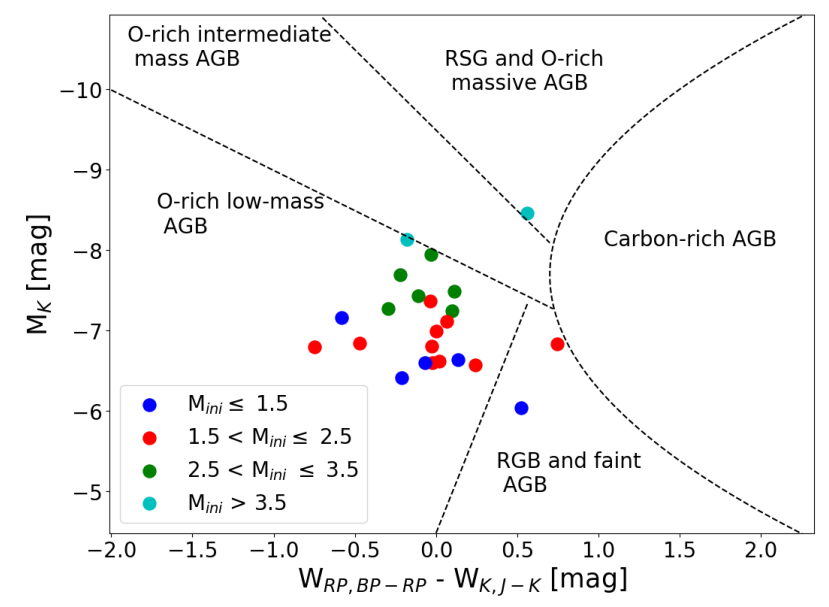

Fig. A.2. The $W_{R P, B P-R P}-W_{K, J-K}$ vs $M_{K}$ diagram of our sample of Tcrich $\mathrm{S}$ stars. The definitions of the boundaries (black dashed lines) are from Lebzelter et al. (2018).

with the abundance from model $\mathrm{A}$ and these differences are listed as columns $\Delta_{B-A}, \ldots, \Delta_{G-A}$ in the bottom panels of Table B.1. Model $\mathrm{H}$ is the one used to compute the error on the abundances of V915 Aql as described in Section 5.8 (or Section 4.4 of S18). The contribution from the atmospheric parameter uncertainties on the error on the $\mathrm{Li}$ and $\mathrm{Tc}$ abundances is given by $\Delta_{H-A}$ in Table B.1 as described in Sect. 5.8

\section{Appendix C: Elemental abundances of sample stars}

In this section we present the tables listing the elemental abundances for the stars of our current study. Table C.1 lists the Tc and $\mathrm{Li}$ abundances in our sample stars and also in Tc-rich stars from S18, S19 and S20 which we computed during the current study. Tables C.2, C.3 and C.4 provide the full list of elemental abundances in our sample stars. Table C.5 lists the different elemental indices [ls/Fe], [hs/Fe], [hs/ls], and [s/Fe] for our sample stars as well as for Tc-rich stars from S18, S19, and S20.
Table B.1. Sensitivity of the $\mathrm{Li}$ and $\mathrm{Tc}$ elemental abundances of V915 Aql upon variations of its atmospheric parameters.

\begin{tabular}{|c|c|c|c|c|c|c|c|}
\hline Model & $\begin{array}{l}T_{\text {eff }} \\
(\mathrm{K})\end{array}$ & $\begin{array}{c}\log g \\
\left(\mathrm{~cm} \mathrm{~s}^{-2}\right)\end{array}$ & $\begin{array}{r}{[\mathrm{Fe} / \mathrm{H}]} \\
(\mathrm{dex})\end{array}$ & $\mathrm{C} / \mathrm{O}$ & $\begin{array}{c}{[\mathrm{s} / \mathrm{Fe}]} \\
(\mathrm{dex})\end{array}$ & \multicolumn{2}{|c|}{$\begin{array}{c}\chi_{t} \\
\left(\mathrm{~km} \mathrm{~s}^{-1}\right)\end{array}$} \\
\hline A & 3400 & 0.0 & -0.5 & 0.75 & 0.00 & & .0 \\
\hline B & 3300 & 0.0 & -0.5 & 0.75 & 0.00 & & .0 \\
\hline $\mathrm{C}$ & 3500 & 0.0 & -0.5 & 0.75 & 0.00 & & .0 \\
\hline $\mathrm{D}$ & 3400 & 1.0 & -0.5 & 0.75 & 0.00 & & .0 \\
\hline $\mathrm{E}$ & 3400 & 0.0 & 0.0 & 0.75 & 0.00 & & .0 \\
\hline $\mathrm{F}$ & 3400 & 0.0 & -0.5 & 0.75 & 0.00 & & .5 \\
\hline G & 3400 & 0.0 & -0.5 & 0.50 & 0.00 & & .0 \\
\hline $\mathrm{H}$ & 3500 & 1.0 & 0.0 & 0.50 & 1.00 & & .0 \\
\hline Element & 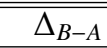 & 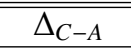 & $\overline{\overline{\Delta_{D-A}}}$ & $\overline{\overline{\Delta_{E-A}}}$ & $\overline{\Delta_{F-A}}$ & $\overline{\overline{\Delta_{G-A}}}$ & $\overline{\Delta_{H-A}}$ \\
\hline$[\mathrm{Li} / \mathrm{Fe}]$ & -0.06 & -0.5 & 0.8 & 0.0 & 0.6 & 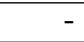 & -0.7 \\
\hline $\log \epsilon_{\mathrm{Tc}}$ & -0.1 & -0.1 & - & -0.1 & -0.2 & -0.2 & -0.5 \\
\hline
\end{tabular}

Notes. The atmospheric parameters of V915 Aql are adopted from S18. A dash in the $\Delta$ column indicates that the agreement between the observed and the synthetic spectra was too poor and that the (unique) line usually providing the abundance for the considered element could not be used for that purpose. Column $\chi_{t}$ corresponds to the microturbulence.

\section{Appendix D: Revised stellar parameters and abundances of stars from S18, S19, S20}

As explained in Sect. 2, the Gaia EDR3 parallaxes became available in the course of our current study. Hence, we revised the stellar parameters of Tc-rich and Tc-poor stars from S18, S19 and S20 using the Gaia EDR3 parallaxes. In Table D.1 we list these revised stellar parameters which were derived using the same method as described in Sect. 4. In Table D.2 we present the re-computed elemental abundances of BD+34 1698 and HD 357941, which are the only two stars for which the EDR3 parallaxes imposed a revision of $\log g$ (see Table D.1). 
Table C.1. Tc and Li abundances of the sample stars as well as of the stars from S18, S19 and S20.

\begin{tabular}{l|rrr}
\hline Star & $\log \epsilon_{\mathrm{Tc}}$ & $\log \epsilon_{\mathrm{Li}}$ & {$[\mathrm{Li} / \mathrm{Fe}]$} \\
\hline HR Peg & -1.3 & 1.3 & 0.6 \\
HD 28833 & -1.2 & $<-0.3$ & $<-1.05$ \\
V812 Oph & 1.0 & - & - \\
AA Cam & -0.4 & $<-0.3$ & $<-1.02$ \\
V1139 Tau & -0.6 & - & - \\
KR CMa & - & - & - \\
CSS 151 & -0.1 & $<-0.6$ & $<-1.4$ \\
CD -275131 & - & - & - \\
BD -18 2608 & -0.1 & $<0.4$ & $<-0.34$ \\
V679 Oph & 0.5 & 0.0 & -0.53 \\
CSS 454 & 0.5 & $<-0.6$ & $<-1.26$ \\
CSS 1152 & 0.5 & 0.0 & -0.91 \\
HD 64147 & -0.1 & $<-0.6$ & $<-0.98$ \\
\hline$o^{1}$ Ori & -0.4 & $<-0.6$ & $<-1.37$ \\
BD +79 156 & -0.7 & $<0.1$ & $<-0.78$ \\
V915 Aql & -0.2 & $<-0.6$ & $<-1.15$ \\
UY Cen & - & -0.3 & -1.35 \\
NQ Pup & -0.3 & $<-0.6$ & $<-1.35$ \\
HD 357941 & -0.6 & $<-0.2$ & $<-0.77$ \\
CSS 154 & -0.6 & $<-0.3$ & $<-1.06$ \\
CSS 182 & 0.0 & $<-0.6$ & $<-1.25$ \\
CD -29 5912 & -0.6 & $<-0.9$ & $<-1.55$ \\
BD +34 1698 & -0.6 & $<-0.2$ & -0.55 \\
\hline
\end{tabular}

Notes. A dash indicates that the agreement between the observed and the synthetic spectra was too poor and that the (unique) line usually providing the abundance for the considered element had to be rejected. The " $<$ " symbol is used when the line is still usable but only provides an upper limit on the abundance.

\section{Appendix E: Spectral windows used for spectral fitting in Sect. 4}

Table E.1 lists the different spectral windows used in the spectral fitting routine to derive an initial estimate of the stellar parameters.

\section{Appendix F: Atomic line list}

Table F.1 lists the lines used for the abundance analysis. 
Table C.2. Elemental abundances of sample stars, along with the standard deviation due to line-to-line scatter.

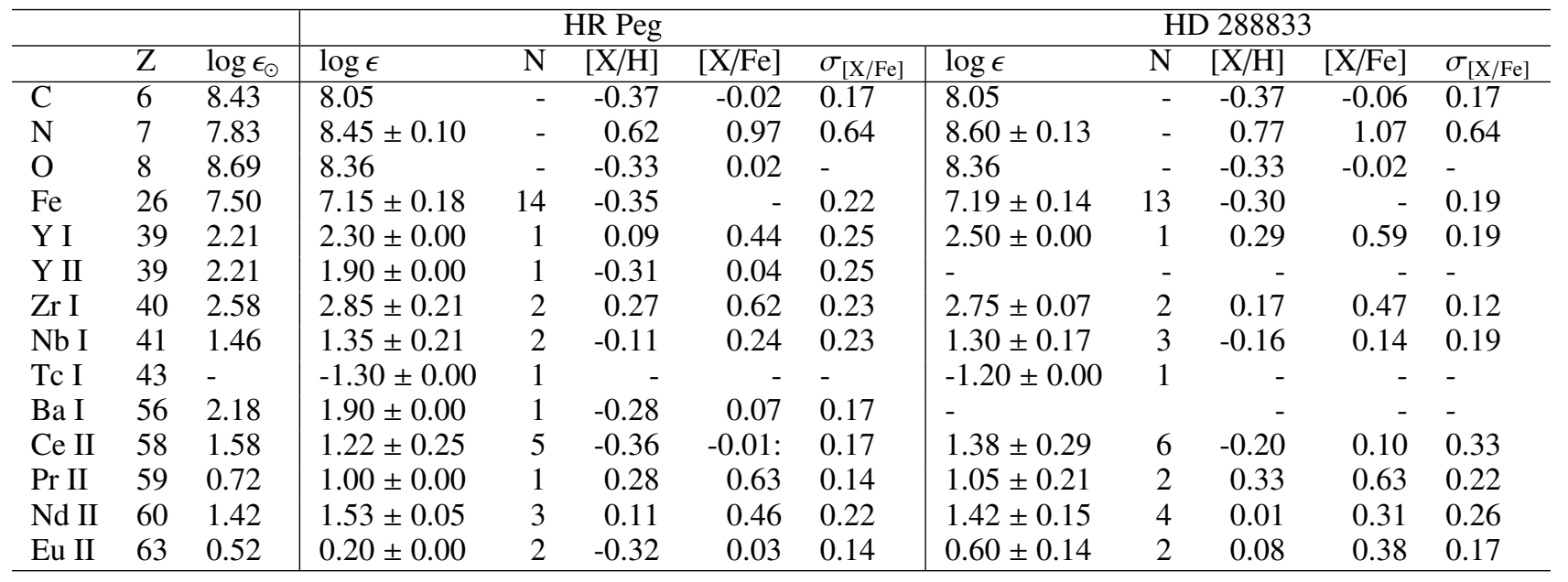

\begin{tabular}{|c|c|c|c|c|c|c|c|c|c|c|c|c|}
\hline & & & \multicolumn{5}{|c|}{ AA Cam } & \multicolumn{5}{|c|}{ V1139 Tau } \\
\hline & $\mathrm{Z}$ & $\log \epsilon_{\odot}$ & $\log \epsilon$ & $\mathrm{N}$ & {$[\mathrm{X} / \mathrm{H}]$} & {$[\mathrm{X} / \mathrm{Fe}]$} & $\sigma_{[\mathrm{X} / \mathrm{Fe}]}$ & $\log \epsilon$ & $\mathrm{N}$ & {$[\mathrm{X} / \mathrm{H}]$} & {$[\mathrm{X} / \mathrm{Fe}]$} & $\sigma_{[\mathrm{X} / \mathrm{Fe}]}$ \\
\hline $\mathrm{C}$ & 6 & 8.43 & 8.06 & - & -0.37 & -0.04 & 0.25 & 8.53 & - & 0.11 & 0.17 & 0.17 \\
\hline $\mathrm{N}$ & 7 & 7.83 & $8.40 \pm 0.13$ & - & 0.57 & 0.89 & 0.65 & $9.90 \pm 0.15$ & - & 2.07 & 2.13 & 0.65 \\
\hline $\mathrm{O}$ & 8 & 8.69 & 8.36 & - & -0.33 & 0.00 & - & 8.66 & & -0.03 & 0.03 & \\
\hline $\mathrm{Fe}$ & 26 & 7.50 & $7.17 \pm 0.12$ & 15 & -0.33 & - & 0.18 & $7.44 \pm 0.13$ & 14 & -0.06 & - & 0.19 \\
\hline Y I & 39 & 2.21 & $1.90 \pm 0.00$ & 1 & -0.31 & 0.02 & 0.22 & - & - & - & - & - \\
\hline Y II & 39 & 2.21 & $2.20 \pm 0.00$ & 1 & -0.01 & 0.32 & 0.22 & - & - & - & - & - \\
\hline Zr I & 40 & 2.58 & $2.75 \pm 0.21$ & 2 & 0.17 & 0.49 & 0.23 & $2.80 \pm 0.00$ & 1 & 0.22 & 0.28 & 0.14 \\
\hline $\mathrm{Nb} \mathrm{I}$ & 41 & 1.46 & $1.30 \pm 0.17$ & 3 & -0.16 & 0.16 & 0.19 & $1.35 \pm 0.21$ & 2 & -0.11 & -0.05 & 0.23 \\
\hline Tc I & 43 & - & $-0.40 \pm 0.00$ & 1 & - & - & - & $-0.60 \pm 0.00$ & 1 & - & - & - \\
\hline $\mathrm{Ba} \mathrm{I}$ & 56 & 2.18 & $1.90 \pm 0.0$ & 1 & -0.28 & 0.05 & 0.17 & $2.40 \pm 0.00$ & 1 & 0.22 & 0.28 & 0.17 \\
\hline Ce II & 58 & 1.58 & $1.24 \pm 0.28$ & 6 & -0.34 & $-0.01:$ & 0.15 & $1.90 \pm 0.10$ & 3 & 0.32 & 0.38 & 0.17 \\
\hline Nd II & 60 & 1.42 & $1.30 \pm 0.17$ & 3 & -0.12 & 0.21 & 0.17 & - & - & - & - & - \\
\hline
\end{tabular}

\begin{tabular}{lll|lrrrr|lrrrr}
\hline & & & \multicolumn{4}{c}{ KR CMa } \\
\hline & $\mathrm{Z}$ & $\log \epsilon_{\odot}$ & $\log \epsilon$ & $\mathrm{N}$ & {$[\mathrm{X} / \mathrm{H}]$} & {$[\mathrm{X} / \mathrm{Fe}]$} & $\sigma_{[\mathrm{X} / \mathrm{Fe}]}$ & $\log \epsilon$ & $\mathrm{N}$ & {$[\mathrm{X} / \mathrm{H}]$} & {$[\mathrm{X} / \mathrm{Fe}]$} & $\sigma_{[\mathrm{X} / \mathrm{Fe}]}$ \\
\hline $\mathrm{C}$ & 6 & 8.43 & 8.36 & - & -0.07 & -0.06 & 0.17 & 8.06 & - & -0.37 & -0.11 & 0.26 \\
$\mathrm{~N}$ & 7 & 7.83 & $8.80 \pm 0.18$ & - & 0.97 & 0.98 & 0.66 & $8.60 \pm 0.12$ & - & 0.77 & 1.03 & 0.65 \\
$\mathrm{O}$ & 8 & 8.69 & 8.66 & - & -0.03 & -0.01 & - & 8.36 & - & -0.33 & -0.07 & \\
$\mathrm{Fe}$ & 26 & 7.50 & $7.48 \pm 0.18$ & 14 & -0.01 & - & 0.22 & $7.24 \pm 0.14$ & 14 & -0.26 & 0 & 0.19 \\
Y I & 39 & 2.21 & $2.80 \pm 0.00$ & 1 & 0.59 & 0.60 & 0.14 & $2.50 \pm 0.00$ & 1 & 0.29 & 0.54 & 0.31 \\
$\mathrm{Zr} \mathrm{I}$ & 40 & 2.58 & - & - & - & - & - & $2.95 \pm 0.21$ & 2 & 0.37 & 0.63 & 0.23 \\
Tc I & 43 & - & - & - & - & - & - & $-0.10 \pm 0.00$ & 1 & - & - & - \\
$\mathrm{Ba}$ I & 56 & 2.18 & $2.50 \pm 0.00$ & 1 & 0.32 & 0.33 & 0.17 & $2.20 \pm 0.00$ & 1 & 0.02 & 0.28 & 0.17 \\
$\mathrm{Ce}$ II & 58 & 1.58 & $1.70 \pm 0.30$ & 4 & 0.12 & 0.13 & 0.22 & $1.81 \pm 0.28$ & 6 & 0.23 & 0.48 & 0.17 \\
Pr II & 59 & 0.72 & - & - & - & - & - & $0.90 \pm 0.00$ & 1 & 0.18 & 0.43 & 0.14 \\
Nd II & 60 & 1.42 & $2.30 \pm 0.00$ & 1 & 0.88 & 0.89 & 0.24 & $1.57 \pm 0.30$ & 4 & 0.15 & 0.41 & 0.37 \\
Sm II & 62 & 0.96 & - & - & - & - & - & $1.10 \pm 0.00$ & 1 & 0.14 & 0.40 & 0.14 \\
Eu II & 63 & 0.52 & - & - & - & - & - & $0.50 \pm 0.00$ & 1 & -0.02 & 0.24 & 0.14 \\
\hline
\end{tabular}

Notes. Solar abundances (third column) are from Asplund et al. (2009). The column labelled $N$ lists the number of lines used to derive the corresponding abundance. The $\sigma_{[\mathrm{X} / \mathrm{Fe}]}$ column lists the total uncertainty on the abundance calculated using the method described in Sect. 5.8 A colon flags an uncertain value. 
Table C.3. Same as Table C.2

\begin{tabular}{lll|lrrrr|lrrrr}
\hline & & & \multicolumn{4}{|c}{$\mathrm{CD}-27^{\circ} 5131$} & \multicolumn{5}{c}{$\mathrm{BD}-18^{\circ} 2608$} \\
\hline & $\mathrm{Z}$ & $\log \epsilon_{\odot}$ & $\log \epsilon$ & $\mathrm{N}$ & {$[\mathrm{X} / \mathrm{H}]$} & {$[\mathrm{X} / \mathrm{Fe}]$} & $\sigma_{[\mathrm{X} / \mathrm{Fe}]}$ & $\log \epsilon$ & $\mathrm{N}$ & {$[\mathrm{X} / \mathrm{H}]$} & {$[\mathrm{X} / \mathrm{Fe}]$} & $\sigma_{[\mathrm{X} / \mathrm{Fe}]}$ \\
\hline $\mathrm{C}$ & 6 & 8.43 & 8.06 & - & -0.37 & -0.07 & 0.26 & 8.23 & - & -0.19 & 0.11 & 0.10 \\
$\mathrm{~N}$ & 7 & 7.83 & $9.60 \pm 0.11$ & - & 1.77 & 2.07 & 0.64 & $8.60 \pm 0.12$ & - & 0.77 & 1.08 & 0.64 \\
$\mathrm{O}$ & 8 & 8.69 & 8.36 & - & -0.33 & -0.03 & - & 8.36 & - & -0.33 & -0.02 & - \\
$\mathrm{Fe}$ & 26 & 7.50 & $7.20 \pm 0.12$ & 10 & -0.30 & - & 0.18 & $7.18 \pm 0.16$ & 13 & -0.31 & - & 0.21 \\
$\mathrm{Sr} I$ & 38 & 2.87 & - & - & - & - & - & $3.00 \pm 0.00$ & 1 & 0.13 & 0.44 & 0.14 \\
$\mathrm{Y} \mathrm{I}$ & 39 & 2.21 & $2.80 \pm 0.00$ & 1 & 0.59 & 0.89 & 0.19 & $2.40 \pm 0.00$ & 1 & 0.19 & 0.50 & 0.4 \\
Y II & 39 & 2.21 & $2.60 \pm 0.00$ & 1 & 0.39 & 0.69 & 0.19 & $2.50 \pm 0.00$ & 1 & 0.29 & 0.60 & 0.40 \\
$\mathrm{Zr} \mathrm{I}$ & 40 & 2.58 & $2.95 \pm 0.21$ & 2 & 0.37 & 0.67 & 0.23 & $3.00 \pm 0.14$ & 2 & 0.42 & 0.73 & 0.17 \\
$\mathrm{Nb}$ I & 41 & 1.46 & $1.20 \pm 0.00$ & 1 & -0.26 & 0.04 & 0.14 & $1.50 \pm 0.00$ & 1 & 0.04 & 0.35 & 0.14 \\
Tc I & 43 & - & - & - & - & - & - & $-0.10 \pm 0.00$ & 1 & - & - & - \\
$\mathrm{Ba}$ I & 56 & 2.18 & $2.80 \pm 0.00$ & 1 & 0.62 & 0.92 & 0.17 & $2.50 \pm 0.00$ & 1 & 0.32 & 0.63 & 0.17 \\
$\mathrm{Ce}$ II & 58 & 1.58 & $1.90 \pm 0.25$ & 7 & 0.32 & 0.62 & 0.25 & $2.14 \pm 0.17$ & 7 & 0.56 & 0.87 & 0.22 \\
Nd II & 60 & 1.42 & $1.80 \pm 0.00$ & 1 & 0.38 & 0.68 & 0.14 & $1.95 \pm 0.21$ & 2 & 0.53 & 0.84 & 0.23 \\
Sm II & 62 & 0.96 & $1.00 \pm 0.00$ & 1 & 0.04 & 0.34 & 0.14 & $1.30 \pm 0.00$ & 2 & 0.34 & 0.65 & 0.14 \\
Eu II & 63 & 0.52 & - & - & - & - & - & $0.50 \pm 0.00$ & 1 & -0.02 & 0.29 & 0.14 \\
\hline
\end{tabular}

\begin{tabular}{|c|c|c|c|c|c|c|c|c|c|c|c|c|}
\hline & & & \multicolumn{5}{|c|}{ V679 Oph } & \multicolumn{5}{|c|}{ V812 Oph } \\
\hline & $\mathrm{Z}$ & $\log \epsilon_{\odot}$ & $\log \epsilon$ & $\mathrm{N}$ & {$[\mathrm{X} / \mathrm{H}]$} & {$[\mathrm{X} / \mathrm{Fe}]$} & $\sigma_{[\mathrm{X} / \mathrm{Fe}]}$ & $\log \epsilon$ & $\mathrm{N}$ & {$[\mathrm{X} / \mathrm{H}]$} & {$[\mathrm{X} / \mathrm{Fe}]$} & $\sigma_{[\mathrm{X} / \mathrm{Fe}]}$ \\
\hline $\bar{C}$ & 6 & 8.43 & 8.31 & - & -0.11 & 0.40 & 0.10 & 8.06 & - & -0.37 & 0.00 & 0.25 \\
\hline $\mathrm{N}$ & 7 & 7.83 & $8.50 \pm 0.09$ & - & 0.67 & 1.19 & 0.64 & $10.10 \pm 0.07$ & - & 2.27 & 2.64 & 0.65 \\
\hline $\mathrm{O}$ & 8 & 8.69 & 8.36 & - & -0.33 & 0.19 & - & 8.36 & - & -0.33 & 0.04 & - \\
\hline $\mathrm{Fe}$ & 26 & 7.50 & $6.98 \pm 0.14$ & 9 & -0.52 & - & 0.19 & $7.12 \pm 0.13$ & 12 & -0.37 & - & 0.19 \\
\hline Sr I & 38 & 2.87 & $3.40 \pm 0.00$ & 1 & 0.53 & 1.05 & 0.14 & - & - & - & - & \\
\hline Y I & 39 & 2.21 & $3.10 \pm 0.00$ & 1 & 0.89 & 1.41 & 0.17 & - & - & - & - & - \\
\hline $\mathrm{Zr} \mathrm{I}$ & 40 & 2.58 & $3.60 \pm 0.34$ & 2 & 1.02 & 1.54 & 0.36 & $3.25 \pm 0.21$ & 2 & 0.67 & 1.04 & 0.23 \\
\hline $\mathrm{Nb} \mathrm{I}$ & 41 & 1.46 & $1.50 \pm 0.00$ & 1 & 0.04 & 0.56 & 0.36 & $1.50 \pm 0.00$ & 1 & 0.04 & 0.41 & 0.14 \\
\hline Tc I & 43 & & $0.50 \pm 0.00$ & 1 & & - & - & $1.00 \pm 0.00$ & 1 & - & - & - \\
\hline $\mathrm{Ba} \mathrm{I}$ & 56 & 2.18 & $3.10 \pm 0.00$ & 1 & 0.92 & 1.44 & 0.17 & $2.80 \pm 0.00$ & 1 & 0.62 & 0.99 & 0.17 \\
\hline Ce II & 58 & 1.58 & $2.35 \pm 0.23$ & 7 & 0.77 & 1.29 & 0.26 & $2.17 \pm 0.25$ & 7 & 0.59 & 0.96 & 0.15 \\
\hline Pr II & 59 & 0.72 & $2.10 \pm 0.00$ & 1 & 1.38 & 1.90 & 0.14 & - & - & - & - & - \\
\hline \multirow[t]{3}{*}{$\mathrm{Nd} \mathrm{II}$} & 60 & 1.42 & $2.40 \pm 0.17$ & 3 & 0.98 & 1.50 & 0.19 & $2.30 \pm 0.34$ & 3 & 0.88 & 1.25 & 0.4 \\
\hline & & & \multicolumn{5}{|c|}{ CSS 454} & \multicolumn{5}{|c|}{ CSS 1152} \\
\hline & $\mathrm{Z}$ & $\log \epsilon_{\odot}$ & $\log \epsilon$ & $\mathrm{N}$ & {$[\mathrm{X} / \mathrm{H}]$} & {$[\mathrm{X} / \mathrm{Fe}]$} & $\sigma_{[\mathrm{X} / \mathrm{Fe}]}$ & $\log \epsilon$ & $\mathrm{N}$ & {$[\mathrm{X} / \mathrm{H}]$} & {$[\mathrm{X} / \mathrm{Fe}]$} & $\sigma_{[\mathrm{X} / \mathrm{Fe}]}$ \\
\hline $\mathrm{C}$ & 6 & 8.43 & 8.06 & - & -0.37 & 0.02 & 0.25 & 8.64 & - & 0.21 & 0.35 & 0.10 \\
\hline $\mathrm{N}$ & 7 & 7.83 & $9.30 \pm 0.14$ & - & 1.47 & 1.86 & 0.65 & $8.60 \pm 0.05$ & - & 0.77 & 0.91 & 0.63 \\
\hline $\mathrm{O}$ & 8 & 8.69 & 8.36 & - & -0.33 & 0.06 & - & 8.66 & - & -0.03 & 0.11 & - \\
\hline $\mathrm{Fe}$ & 26 & 7.50 & $7.11 \pm 0.14$ & 12 & -0.39 & - & 0.19 & $7.36 \pm 0.13$ & 6 & -0.14 & - & 0.19 \\
\hline Y I & 39 & 2.21 & $2.50 \pm 0.00$ & 1 & 0.29 & 0.68 & 0.36 & $3.40 \pm 0.00$ & 1 & 1.19 & 1.33 & 0.17 \\
\hline Y II & 39 & 2.21 & $2.50 \pm 0.00$ & 1 & 0.29 & 0.68 & 0.36 & - & - & - & - & - \\
\hline $\mathrm{Zr} \mathrm{I}$ & 40 & 2.58 & $3.15 \pm 0.07$ & 2 & 0.57 & 0.96 & 0.12 & $3.75 \pm 0.07$ & 2 & 1.17 & 1.31 & 0.12 \\
\hline $\mathrm{Nb} \mathrm{I}$ & 41 & 1.46 & $1.20 \pm 0.00$ & 1 & -0.26 & -0.26 & 0.13 & $2.10 \pm 0.00$ & 1 & 0.64 & 0.78 & 0.14 \\
\hline Tc I & 43 & & $0.50 \pm 0.00$ & 1 & & - & - & $0.50 \pm 0.00$ & 1 & - & - & - \\
\hline $\mathrm{Ba} \mathrm{I}$ & 56 & 2.18 & $2.80 \pm 0.00$ & 1 & 0.62 & 1.01 & 0.17 & $3.10 \pm 0.00$ & 1 & 0.92 & 1.06 & 0.17 \\
\hline Ce II & 58 & 1.58 & $2.17 \pm 0.21$ & 8 & 0.59 & 0.98 & 0.25 & $2.66 \pm 0.22$ & 3 & 1.08 & 1.22 & 0.24 \\
\hline Pr II & 59 & 0.72 & $1.00 \pm 0.00$ & 1 & 0.28 & 0.67 & 0.14 & - & - & - & - & - \\
\hline $\mathrm{Nd}$ II & 60 & 1.42 & $2.10 \pm 0.00$ & 1 & 0.68 & 1.07 & 0.14 & $2.75 \pm 0.07$ & 2 & 1.33 & 1.47 & 0.12 \\
\hline Sm II & 62 & 0.96 & $1.50 \pm 0.00$ & 1 & 0.54 & 0.93 & 0.14 & - & - & - & - & - \\
\hline Eu II & 63 & 0.52 & $0.55 \pm 0.07$ & 2 & 0.03 & 0.42 & 0.12 & - & - & - & - & - \\
\hline
\end{tabular}


Table C.4. Same as Tables C.2 and C.3

\begin{tabular}{lll|lrrrl}
\hline & & & \multicolumn{5}{|c}{$\mathrm{HD} 64147$} \\
\hline & $\mathrm{Z}$ & $\log \epsilon_{\odot}$ & $\log \epsilon$ & $\mathrm{N}$ & {$[\mathrm{X} / \mathrm{H}]$} & {$[\mathrm{X} / \mathrm{Fe}]$} & $\sigma_{[\mathrm{X} / \mathrm{Fe}]}$ \\
\hline $\mathrm{C}$ & 6 & 8.43 & 8.06 & - & -0.37 & 0.30 & 0.17 \\
$\mathrm{~N}$ & 7 & 7.83 & $8.00 \pm 0.07$ & - & 0.17 & 0.84 & 0.64 \\
$\mathrm{O}$ & 8 & 8.69 & 8.36 & - & -0.33 & 0.34 & - \\
$\mathrm{Fe}$ & 26 & 7.50 & $6.82 \pm 0.10$ & 11 & -0.67 & - & 0.17 \\
$\mathrm{Y} \mathrm{I}$ & 39 & 2.21 & $1.90 \pm 0.00$ & 1 & -0.31 & 0.36 & 0.17 \\
$\mathrm{Y} \mathrm{II}$ & 39 & 2.21 & $1.90 \pm 0.00$ & 1 & -0.31 & 0.36 & 0.17 \\
$\mathrm{Zr} \mathrm{I}$ & 40 & 2.58 & $2.45 \pm 0.21$ & 2 & -0.13 & 0.54 & 0.23 \\
$\mathrm{Nb}$ I & 41 & 1.46 & $1.05 \pm 0.21$ & 2 & -0.41 & 0.26 & 0.23 \\
$\mathrm{Tc}$ I & 43 & & $-0.10 \pm 0.00$ & 1 & - & - & - \\
$\mathrm{Ba}$ I & 56 & 2.18 & $1.90 \pm 0.00$ & 1 & -0.28 & 0.39 & 0.17 \\
$\mathrm{Ce}$ II & 58 & 1.58 & $1.21 \pm 0.20$ & 7 & -0.37 & 0.30 & 0.24 \\
$\mathrm{Pr}$ II & 59 & 0.72 & $0.40 \pm 0.00$ & 1 & -0.32 & 0.35 & 0.14 \\
$\mathrm{Nd}$ II & 60 & 1.42 & $1.30 \pm 0.14$ & 2 & -0.12 & 0.55 & 0.17 \\
$\mathrm{Sm} \mathrm{II}$ & 62 & 0.96 & $0.75 \pm 0.07$ & 2 & -0.21 & 0.46 & 0.12 \\
Eu II & 63 & 0.52 & $0.20 \pm 0.00$ & 1 & -0.32 & 0.35 & 0.14 \\
\hline
\end{tabular}

Table C.5. The heavy (hs) and light (1s) s-process indices of the sample stars.

\begin{tabular}{l|cccccc}
\hline Star & {$[\mathrm{ls} / \mathrm{Fe}]$} & {$[\mathrm{hs} / \mathrm{Fe}]$} & {$[\mathrm{hs} / \mathrm{ls}]$} & $\sigma_{[\mathrm{hs} / \mathrm{ls}]}$ & {$[\mathrm{s} / \mathrm{Fe}]$} & $\sigma_{[\mathrm{s} / \mathrm{Fe}]}$ \\
\hline HR Peg & 0.43 & 0.26 & -0.16 & 0.27 & 0.31 & 0.31 \\
HD 288833 & 0.53 & - & - & - & - & - \\
V812 Oph & - & 1.12 & - & - & - & - \\
AA Cam & 0.33 & 0.12 & -0.20 & 0.32 & 0.24 & 0.36 \\
V1139 Tau & - & - & - & - & - & - \\
KR CMa & - & 0.61 & - & - & - & - \\
CSS 151 & 0.58 & 0.34 & -0.24 & 0.38 & 0.48 & 0.42 \\
CD -27 5131 & 0.73 & 0.80 & 0.07 & 0.26 & 0.79 & 0.35 \\
BD -18 2608 & 0.64 & 0.73 & 0.09 & 0.26 & 0.63 & 0.29 \\
V679 Oph & 1.47 & 1.47 & -0.01 & 0.32 & 1.46 & 0.43 \\
CSS 454 & 0.82 & 1.04 & 0.22 & 0.21 & 0.88 & 0.27 \\
CSS 1152 & 1.32 & 1.26 & -0.05 & 0.20 & 1.23 & 0.27 \\
HD 64147 & 0.45 & 0.47 & 0.02 & 0.26 & 0.43 & 0.33 \\
\hline$o^{1}$ Ori & 0.16 & -0.02 & -0.19 & 0.30 & 0.01 & 0.36 \\
BD +79 156 & 0.80 & 0.72 & -0.08 & 0.21 & 0.73 & 0.27 \\
V915 Aql & 0.28 & 0.47 & 0.19 & 0.27 & 0.36 & 0.37 \\
UY Cen & 1.12 & 1.07 & -0.04 & 0.57 & 0.92 & 0.85 \\
NQ Pup & 0.44 & 0.46 & 0.02 & 0.40 & 0.40 & 0.46 \\
HD 357941 & 0.21 & - & - & - & 0.24 & 0.75 \\
CSS 154 & 0.44 & - & - & - & 0.40 & 0.87 \\
CSS 182 & 0.63 & 0.70 & 0.07 & 0.62 & 0.66 & 0.82 \\
CD -29 5912 & 0.66 & 0.82 & 0.17 & 0.56 & 0.67 & 0.75 \\
BD+34 1698 & 0.80 & 0.9 & 0.09 & 0.40 & 0.87 & 0.59 \\
\hline
\end{tabular}

Notes. The $[\mathrm{ls} / \mathrm{Fe}]$ index has been derived using $\mathrm{Y}$ and $\mathrm{Zr}$, whereas the $[\mathrm{hs} / \mathrm{Fe}]$ index relies on $\mathrm{Ba}$ and $\mathrm{Nd}$ abundances. The error on the $[\mathrm{hs} / \mathrm{ls}]$ ratio was computed by quadratically adding the error on the individual abundances of the elements considered to compute the $[\mathrm{hs} / \mathrm{Fe}]$ and $[\mathrm{ls} / \mathrm{Fe}] \mathrm{ratios}$. The same method is used to estimate the error on the $[\mathrm{s} / \mathrm{Fe}]$ ratio, which is based on the abundances of $\mathrm{Y}, \mathrm{Zr}$ and $\mathrm{Ba}$. 
Table D.1. Atmospheric parameters for S stars from S18, S19 and S20. The $L$ column indicates the luminosity and its error due to the Gaia EDR3 error on the parallax. All the other columns are the same as in Table 3

\begin{tabular}{|c|c|c|c|c|c|c|c|c|c|}
\hline Name & $\begin{array}{l}T_{\text {eff }} \\
(\mathrm{K})\end{array}$ & $\begin{array}{c}L \\
\left(L_{\odot}\right)\end{array}$ & $\log g$ & {$[\mathrm{Fe} / \mathrm{H}]$} & $\sigma_{[\mathrm{Fe} / \mathrm{H}]}$ & $\mathrm{C} / \mathrm{O}$ & {$[\mathrm{s} / \mathrm{Fe}]$} & $\begin{array}{l}\mathrm{M}_{\text {curr }} \\
\left(M_{\odot}\right)\end{array}$ & $\begin{array}{l}\mathrm{M}_{\mathrm{ini}} \\
\left(M_{\odot}\right)\end{array}$ \\
\hline \multicolumn{10}{|c|}{ Tc-rich S stars from S18 } \\
\hline V915 Aql & $\begin{array}{c}3400 \\
(3400 ; 3400)\end{array}$ & $\begin{array}{c}2000 \\
(1900 ; 2000)\end{array}$ & $\begin{array}{c}0 \\
(0 ; 1)\end{array}$ & -0.50 & 0.15 & $\begin{array}{c}0.75 \\
(0.65 ; 0.75)\end{array}$ & $\begin{array}{c}0 \\
(0 ; 1)\end{array}$ & 0.8 & 1.0 \\
\hline NQ Pup & $\begin{array}{c}3700 \\
(3500 ; 3700)\end{array}$ & $\begin{array}{c}2800 \\
(2700 ; 2900)\end{array}$ & $\begin{array}{c}1 \\
(1 ; 2)\end{array}$ & -0.30 & 0.05 & $\begin{array}{c}0.50 \\
(0.5 ; 0.75)\end{array}$ & $\begin{array}{c}1 \\
(1 ; 1)\end{array}$ & 2.4 & 2.5 \\
\hline UY Cen & $\begin{array}{c}3300 \\
(3000 ; 3400)\end{array}$ & $\begin{array}{c}11884 \\
(11100 ; 13000)\end{array}$ & $\begin{array}{c}0 \\
(0 ; 3)\end{array}$ & -0.30 & 0.15 & $\begin{array}{c}0.999 \\
(0.971 ; 0.999)\end{array}$ & $\begin{array}{c}1 \\
(1 ; 2)\end{array}$ & 3.6 & 4.0 \\
\hline \multicolumn{10}{|c|}{ Tc-rich S stars from S19 } \\
\hline HD 357941 & $\begin{array}{c}3400 \\
(100 ; 100)\end{array}$ & $\begin{array}{c}8300 \\
(7400 ; 9300)\end{array}$ & $\begin{array}{c}0 \\
(0 ; 1)\end{array}$ & -0.48 & 0.14 & $\begin{array}{c}0.5 \\
(0.5 ; 0.75)\end{array}$ & $\begin{array}{c}0 \\
(0 ; 1)\end{array}$ & 3.2 & 3.5 \\
\hline CSS 154 & $\begin{array}{c}3400 \\
(100 ; 100)\end{array}$ & $\begin{array}{c}3000 \\
(2700 ; 3300)\end{array}$ & $\begin{array}{c}1 \\
(1 ; 3)\end{array}$ & -0.29 & 0.20 & $\begin{array}{c}0.5 \\
(0.5 ; 0.899)\end{array}$ & $\begin{array}{c}0 \\
(0 ; 1)\end{array}$ & 1.4 & 1.6 \\
\hline CSS 182 & $\begin{array}{c}3500 \\
(100 ; 100)\end{array}$ & $\begin{array}{c}2500 \\
(2200 ; 2800)\end{array}$ & $\begin{array}{c}1 \\
(1 ; 3)\end{array}$ & -0.40 & 0.21 & $\begin{array}{c}0.5 \\
(0.5 ; 0.899)\end{array}$ & $\begin{array}{c}1 \\
(1 ; 1)\end{array}$ & 1.1 & 1.3 \\
\hline $\mathrm{CD}-29^{\circ} 5912$ & $\begin{array}{c}3600 \\
(100 ; 100)\end{array}$ & $\begin{array}{c}2700 \\
(2500 ; 3000)\end{array}$ & $\begin{array}{c}1 \\
(1 ; 3)\end{array}$ & -0.40 & 0.22 & $\begin{array}{c}0.5 \\
(0.5 ; 0.899)\end{array}$ & $\begin{array}{c}1 \\
(1 ; 1)\end{array}$ & 1.3 & 1.5 \\
\hline $\mathrm{BD}+34^{\circ} 1698$ & $\begin{array}{c}3400 \\
(200 ; 200)\end{array}$ & $\begin{array}{c}3800 \\
(3300 ; 4400)\end{array}$ & $\begin{array}{c}0 \\
(0 ; 3)\end{array}$ & -0.70 & 0.20 & $\begin{array}{c}0.5 \\
(0.5 ; 0.899)\end{array}$ & $\begin{array}{c}1 \\
(1 ; 1)\end{array}$ & 1.2 & 1.4 \\
\hline \multicolumn{10}{|c|}{ Tc-rich S stars from $\mathrm{S} 20$} \\
\hline$o^{1}$ Ori & $\begin{array}{c}3500 \\
(3500 ; 3600)\end{array}$ & $\begin{array}{c}3000 \\
(2800 ; 3300)\end{array}$ & $\begin{array}{c}1 \\
(0 ; 2)\end{array}$ & -0.28 & 0.19 & $\begin{array}{c}0.75 \\
(0.5 ; 0.90)\end{array}$ & $\begin{array}{c}0 \\
(0 ; 1)\end{array}$ & 1.6 & 1.8 \\
\hline $\mathrm{BD}+79156$ & $\begin{array}{c}3600 \\
(3600 ; 3700)\end{array}$ & $\begin{array}{c}2600 \\
(2400 ; 2700)\end{array}$ & $\begin{array}{c}1 \\
(1 ; 3)\end{array}$ & -0.16 & 0.12 & $\begin{array}{c}0.50 \\
(0.50 ; 0.75)\end{array}$ & $\begin{array}{c}1 \\
(1 ; 1)\end{array}$ & 2 & 2.1 \\
\hline \multicolumn{10}{|c|}{ Tc-poor S stars from S18 } \\
\hline HD 189581 & $\begin{array}{c}3500 \\
(3500 ; 3500)\end{array}$ & $\begin{array}{c}1700 \\
(1600 ; 1800)\end{array}$ & $\begin{array}{c}1 \\
(1 ; 2)\end{array}$ & 0.00 & 0.13 & $\begin{array}{c}0.50 \\
(0.50 ; 0.75)\end{array}$ & $\begin{array}{c}03 \\
(0 ; 0)\end{array}$ & 1.6 & 1.8 \\
\hline HD 233158 & $\begin{array}{c}3600 \\
(3600 ; 3600)\end{array}$ & $\begin{array}{c}1900 \\
(1800 ; 2000)\end{array}$ & $\begin{array}{c}1 \\
(1 ; 1)\end{array}$ & -0.40 & 0.16 & $\begin{array}{c}0.50 \\
(0.50 ; 0.75)\end{array}$ & $\begin{array}{c}1 \\
(1 ; 1)\end{array}$ & 1.1 & 1.3 \\
\hline HD 191589 & $\begin{array}{c}3700 \\
(3700 ; 3800)\end{array}$ & $\begin{array}{c}500 \\
(500 ; 600)\end{array}$ & $\begin{array}{c}1 \\
(1 ; 2)\end{array}$ & -0.30 & 0.10 & $\begin{array}{c}0.75 \\
(0.75 ; 0.75)\end{array}$ & $\begin{array}{c}1 \\
(1 ; 1)\end{array}$ & 1 & 1 \\
\hline HD 191226 & $\begin{array}{c}3600 \\
(3600 ; 3600)\end{array}$ & $\begin{array}{c}6400 \\
(6100 ; 6800)\end{array}$ & $\begin{array}{c}1 \\
(0 ; 1)\end{array}$ & -0.10 & 0.13 & $\begin{array}{c}0.75 \\
(0.50 ; 0.899)\end{array}$ & $\begin{array}{c}1 \\
(1 ; 1)\end{array}$ & 4.6 & 5.0 \\
\hline V530 Lyr & $\begin{array}{c}3500 \\
(3500 ; 3600)\end{array}$ & $\begin{array}{c}1700 \\
(1600 ; 1800)\end{array}$ & $\begin{array}{c}1 \\
(1 ; 3)\end{array}$ & 0.00 & 0.10 & $\begin{array}{c}0.50 \\
(0.50 ; 0.75)\end{array}$ & $\begin{array}{c}1 \\
(1 ; 1)\end{array}$ & 1.6 & 1.8 \\
\hline HD 215336 & $\begin{array}{c}3700 \\
(3700 ; 3700)\end{array}$ & $\begin{array}{c}600 \\
(500 ; 600)\end{array}$ & $\begin{array}{c}1 \\
(1 ; 1)\end{array}$ & 0.00 & 0.12 & $\begin{array}{c}0.50 \\
(0.50 ; 0.75)\end{array}$ & $\begin{array}{c}1 \\
(1 ; 1)\end{array}$ & 1.0 & 1.2 \\
\hline HD 150922 & $\begin{array}{c}3600 \\
(3600 ; 3600)\end{array}$ & $\begin{array}{c}5000 \\
(4800 ; 5100)\end{array}$ & $\begin{array}{c}0 \\
(0 ; 1)\end{array}$ & -0.50 & 0.12 & $\begin{array}{c}0.50 \\
(0.50 ; 0.75)\end{array}$ & $\begin{array}{c}1 \\
(1 ; 1)\end{array}$ & 2.1 & 2.2 \\
\hline HD 63733 & $\begin{array}{c}3700 \\
(3700 ; 3700)\end{array}$ & $\begin{array}{c}1500 \\
(1400 ; 1600)\end{array}$ & $\begin{array}{c}1 \\
(1 ; 2)\end{array}$ & -0.10 & 0.13 & $\begin{array}{c}0.50 \\
(0.50 ; 0.75)\end{array}$ & $\begin{array}{c}1 \\
(1 ; 1)\end{array}$ & 2.2 & 2.3 \\
\hline $\mathrm{BD}+69^{\circ} 524$ & $\begin{array}{c}3600 \\
(3600 ; 3600)\end{array}$ & $\begin{array}{c}900 \\
(800 ; 900)\end{array}$ & $\begin{array}{c}1 \\
(1 ; 2)\end{array}$ & -0.40 & 0.11 & $\begin{array}{c}0.50 \\
(0.50 ; 0.75)\end{array}$ & $\begin{array}{c}0 \\
(0 ; 0)\end{array}$ & 1.0 & 1.0 \\
\hline $\mathrm{BD}+28^{\circ} 4592$ & $\begin{array}{c}3700 \\
(3700 ; 3800)\end{array}$ & $\begin{array}{c}600 \\
(600 ; 700)\end{array}$ & $\begin{array}{c}1 \\
(1 ; 2)\end{array}$ & -0.10 & 0.12 & $\begin{array}{c}0.75 \\
(0.50 ; 0.899)\end{array}$ & $\begin{array}{c}1 \\
(1 ; 1)\end{array}$ & 1.1 & 1.3 \\
\hline V1135 Tau & $\begin{array}{c}3400 \\
(3400 ; 3500)\end{array}$ & $\begin{array}{c}1700 \\
(1600 ; 1800)\end{array}$ & $\begin{array}{c}1 \\
(0 ; 1)\end{array}$ & -0.20 & 0.14 & $\begin{array}{c}0.50 \\
(0.5 ; 0.951)\end{array}$ & $\begin{array}{c}1 \\
(1 ; 1)\end{array}$ & 1.0 & 1.2 \\
\hline $\mathrm{AB} \mathrm{Col}$ & $\begin{array}{c}3500 \\
(3300 ; 3500)\end{array}$ & $\begin{array}{c}2000 \\
(1900 ; 2000)\end{array}$ & $\begin{array}{c}1 \\
(1 ; 2)\end{array}$ & 0.00 & 0.14 & $\begin{array}{c}0.50 \\
(0.50 ; 0.75)\end{array}$ & $\begin{array}{c}1 \\
(1 ; 1)\end{array}$ & 1.9 & 2.0 \\
\hline TYC 5971-534-1 & $\begin{array}{c}3600 \\
(3600 ; 3600)\end{array}$ & $\begin{array}{c}800 \\
(700 ; 900)\end{array}$ & $\begin{array}{c}1 \\
(0 ; 2)\end{array}$ & -0.10 & 0.18 & $\begin{array}{c}0.899 \\
(0.50 ; 0.899)\end{array}$ & $\begin{array}{c}1 \\
(1 ; 2)\end{array}$ & 1.1 & 1.3 \\
\hline $\mathrm{BD}-10^{\circ} 1977$ & $\begin{array}{c}3500 \\
(3500 ; 3600)\end{array}$ & $\begin{array}{c}6100 \\
(5700 ; 6500)\end{array}$ & $\begin{array}{c}0 \\
(0 ; 1)\end{array}$ & -0.50 & 0.15 & $\begin{array}{c}0.50 \\
(0.50 ; 0.899)\end{array}$ & $\begin{array}{c}1 \\
(1 ; 1)\end{array}$ & 1.5 & 1.7 \\
\hline FX CMa & $\begin{array}{c}3500 \\
(3500 ; 3500)\end{array}$ & $\begin{array}{c}6500 \\
(6100 ; 7100)\end{array}$ & $\begin{array}{c}1 \\
(1 ; 1)\end{array}$ & 0.00 & 0.14 & $\begin{array}{c}0.971 \\
(0.951 ; 0.971)\end{array}$ & $\begin{array}{c}1 \\
(1 ; 1)\end{array}$ & 4.2 & 4.5 \\
\hline BD- $22^{\circ} 1742$ & $\begin{array}{c}4000 \\
(4000 ; 4000)\end{array}$ & $\begin{array}{c}600 \\
(500 ; 600)\end{array}$ & $\begin{array}{c}1 \\
(1 ; 5)\end{array}$ & -0.30 & 0.09 & $\begin{array}{c}0.75 \\
(0.50 ; 0.899)\end{array}$ & $\begin{array}{c}0 \\
(0 ; 1)\end{array}$ & 1.5 & 1.7 \\
\hline
\end{tabular}


Table D.2. Elemental abundances for the sample stars, along with the standard deviation due to line-to-line scatter. Solar abundances (third column) are from Asplund et al. (2009). The column labelled $N$ lists the number of lines used to derive the abundance. The $\sigma_{[\mathrm{X} / \mathrm{Fe}]}$ column lists the total uncertainty on the abundances calculated using the method described in Sect. 5.8. The abundances and total error budget for V915 Aql were retrieved from S19.

\begin{tabular}{lcc|lrrrr|lrrrr}
\hline & & & \multicolumn{4}{c}{$\mathrm{BD}+34^{\circ} 1698$} & \multicolumn{4}{c}{$\mathrm{HD} 357941$} \\
\hline & $Z$ & $\log \epsilon_{\odot}^{a}$ & $\log \epsilon$ & $\mathrm{N}$ & {$[\mathrm{X} / \mathrm{H}]$} & {$[\mathrm{X} / \mathrm{Fe}]$} & $\sigma_{[\mathrm{X} / \mathrm{Fe}]}$ & $\log \epsilon$ & $\mathrm{N}$ & {$[\mathrm{X} / \mathrm{H}]$} & {$[\mathrm{X} / \mathrm{Fe}]$} & $\sigma_{[\mathrm{X} / \mathrm{Fe}]}$ \\
\hline $\mathrm{C}$ & 6 & 8.43 & 8.059 & - & -0.371 & 0.329 & - & 8.059 & - & -0.371 & 0.109 & - \\
$\mathrm{N}$ & 7 & 7.83 & 9.0 & - & 1.17 & 1.87 & - & 8.0 & - & 0.17 & 0.65 & - \\
$\mathrm{O}$ & 8 & 8.69 & 8.36 & - & -0.33 & 0.37 & - & 8.36 & - & -0.33 & 0.15 & - \\
$\mathrm{Fe}$ & 26 & 7.5 & $6.80 \pm 0.27$ & 13 & -0.70 & - & 0.3 & $7.02 \pm 0.14$ & 12 & -0.48 & - & 0.2 \\
$\mathrm{Y} \mathrm{I}$ & 39 & 2.21 & $2.50 \pm 0.1$ & 1 & -0.11 & 0.59 & 0.2 & $2.10 \pm 0.00$ & 1 & -0.11 & 0.37 & 0.5 \\
$\mathrm{Y} \mathrm{II}$ & 39 & 2.21 & $2.20 \pm 0.1$ & 1 & -0.01 & 0.69 & 0.2 & $2.00 \pm 0.00$ & 1 & -0.21 & 0.27 & 0.5 \\
$\mathrm{Zr} \mathrm{I}$ & 40 & 2.58 & $2.65 \pm 0.07$ & 2 & 0.07 & 0.77 & 0.4 & $2.20 \pm 0.21$ & 2 & -0.38 & 0.10 & 0.4 \\
$\mathrm{Nb}$ I & 41 & 1.46 & $1.50 \pm 0.00$ & 1 & 0.04 & 0.74 & 0.3 & $1.2 \pm 0.00$ & 2 & -0.26 & 0.22 & 0.3 \\
$\mathrm{Ba}$ I & 56 & 2.18 & $2.5 \pm 0.00$ & 1 & 0.29 & 0.99 & 0.4 & $2.0 \pm 0.00$ & 1 & -0.18 & 0.30 & 0.4 \\
$\mathrm{Ce}$ II & 58 & 1.58 & $1.24 \pm 0.13$ & 5 & -0.34 & 0.36 & 0.3 & $1.07 \pm 0.30$ & 4 & -0.51 & -0.03 & 0.3 \\
\hline
\end{tabular}


Table E.1. The spectral windows used to compare observed spectra with MARCS synthetic spectra of S stars. The band and normalisation limits are expressed in nm.

\begin{tabular}{|c|c|c|c|}
\hline \multirow{2}{*}{\multicolumn{2}{|c|}{ Band }} & $\sin$ & $\lambda_{\max }$ \\
\hline & & \multicolumn{2}{|c|}{ Normalisation } \\
\hline 400.0 & 420.0 & 408.0 & 412.0 \\
\hline 420.0 & 440.0 & 432.0 & 434.0 \\
\hline 440.0 & 458.0 & 456.0 & 458.0 \\
\hline 458.0 & 480.0 & 472.0 & 476.0 \\
\hline 480.0 & 490.0 & 489.4 & 489.9 \\
\hline 490.0 & 495.0 & 494.0 & 495.0 \\
\hline 495.0 & 516.0 & 515.5 & 516.0 \\
\hline 516.0 & 530.0 & 516.0 & 516.3 \\
\hline 530.0 & 544.0 & 543.0 & 544.5 \\
\hline 544.0 & 565.0 & 560.5 & 562.0 \\
\hline 565.0 & 575.5 & 573.0 & 573.7 \\
\hline 575.5 & 588.0 & 580.0 & 582.0 \\
\hline 588.0 & 615.0 & 613.0 & 615.0 \\
\hline 615.0 & 647.0 & 645.0 & 647.0 \\
\hline 647.0 & 671.5 & 670.0 & 671.5 \\
\hline 671.5 & 685.0 & 683.0 & 685.0 \\
\hline 703.0 & 720.0 & 703.0 & 705.0 \\
\hline
\end{tabular}


Table F.1. Atomic lines used in this study.

\begin{tabular}{|c|c|c|c|c|}
\hline Species & $\lambda[\AA]$ & $\chi[\mathrm{eV}]$ & $\log g f$ & Reference \\
\hline \multirow[t]{24}{*}{ Fe I } & 7386.334 & 4.913 & -0.268 & Kurucz (2007) \\
\hline & 7389.398 & 4.301 & -0.460 & Kurucz (2007) \\
\hline & 7418.667 & 4.143 & -1.376 & O'Brian et al. (1991) \\
\hline & 7421.554 & 4.638 & -1.800 & Martin et al.(1988) \\
\hline & 7443.022 & 4.186 & -1.820 & Martin et al.(1988) \\
\hline & 7461.263 & 5.507 & -3.059 & Kurucz (2007) \\
\hline & 7498.530 & 4.143 & -2.250 & Martın et al.(1988) \\
\hline & 7540.430 & 2.727 & -3.850 & 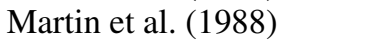 \\
\hline & 7568.899 & 4.283 & -0.773 & Kurucz (2007) \\
\hline & 7583.787 & 3.018 & -1.885 & O'Brian et al. (1991) \\
\hline & 7586.018 & 4.313 & -0.458 & Kurucz (2007) \\
\hline & 7832.196 & 4.435 & 0.111 & Kurucz, (2007) \\
\hline & 7937.139 & 4.313 & 0.225 & $\overline{\text { Kurucz }}(\overline{\overline{2007}})$ \\
\hline & 8108.320 & 2.728 & -3.898 & Kurucz (2007) \\
\hline & 8239.127 & 2.424 & -3.180 & O'Brian et al. (1991) \\
\hline & 8248.129 & 4.371 & -0.887 & Kurucz (2007) \\
\hline & 8621.601 & 2.949 & -2.320 & Kurucz (2007) \\
\hline & 8616.280 & 4.913 & -0.655 & Nave et al. (1994) \\
\hline & 8698.706 & 2.990 & -3.452 & Kurucz (2007) \\
\hline & 8699.454 & 4.955 & -0.380 & Nave et al.(1994) \\
\hline & 8710.404 & 5.742 & -5.156 & Kurucz, 2007$)$ \\
\hline & 8729.144 & 3.415 & -2.871 & Kurucz (2007) \\
\hline & 8747.425 & 3.018 & -3.176 & Kurucz $(\overline{2007})$ \\
\hline & 8763.966 & 4.652 & -0.146 & Nave et al. (1994) \\
\hline \multirow[t]{2}{*}{ Sr I } & 4872.488 & 1.798 & -0.060 & Kupka et al.(1999) \\
\hline & 7070.070 & 1.847 & -0.030 & García \& Campos 1988) \\
\hline Y I & 8800.588 & 0.000 & -2.240 & Corliss \& Bozman (1962) \\
\hline Y II & 7881.881 & 1.839 & -0.570 & Nilsson et al. (1991) \\
\hline \multirow[t]{2}{*}{$\mathrm{Zr} \mathrm{I}$} & 7819.374 & 1.822 & -0.380 & Biémont et al. (1981) \\
\hline & 7849.365 & 0.687 & -1.300 & Biémont et al. (1981) \\
\hline \multirow[t]{3}{*}{$\mathrm{Nb} \mathrm{I}$} & 5189.186 & 0.130 & -1.394 & Duquette \& Lawler (1982) \\
\hline & 5271.524 & 0.142 & -1.240 & Duquette \& Lawler \\
\hline & 5350.722 & 0.267 & -0.862 & Duquette \& Lawler \\
\hline \multirow[t]{3}{*}{ Tc I } & 4238.190 & 0.000 & -0.550 & Palmeri et al. (2005) \\
\hline & 4262.270 & 0.000 & -0.350 & Palmeri et al. (2005) \\
\hline & 4297.060 & 0.000 & -0.190 & Palmeri et al. (2005) \\
\hline $\mathrm{Ba} \mathrm{I}$ & 7488.077 & 1.190 & -0.230 & Miles \& Wiese (1969) \\
\hline \multirow[t]{8}{*}{ Ce II } & 7580.913 & 0.327 & -2.120 & Palmeri et al. $(2000)$ \\
\hline & 8025.571 & 0.000 & -1.420 & Meggers et al. (1975) \\
\hline & 8394.514 & 0.265 & -2.590 & Palmeri et al. $(2000)$ \\
\hline & 8404.133 & 0.704 & -1.670 & Palmeri et al. $(2000)$ \\
\hline & 8405.254 & 0.295 & -2.100 & Meggers et al. (1975) \\
\hline & 8716.659 & 0.122 & -1.980 & Meggers et al. (1975) \\
\hline & 8769.913 & 0.553 & -2.370 & Palmeri et al. $(2000)$ \\
\hline & 8772.135 & 0.357 & -1.260 & Palmer1 et al. (2000) \\
\hline \multirow[t]{6}{*}{$\mathrm{Nd}$ II } & 4715.586 & 0.205 & -0.900 & Den Hartog et al. (2003) \\
\hline & 5276.869 & 0.859 & -0.440 & Meggers et al.(1975) \\
\hline & 5293.160 & 0.823 & 0.100 & Den Hartog et al. (2003) \\
\hline & 5319.810 & 0.550 & -0.140 & Den Hartog et al. $(\overline{2003})$ \\
\hline & 5385.888 & 0.742 & -0.860 & Meggers et al. (1975) \\
\hline & 7513.736 & 0.933 & -1.241 & Meggers et al. $(\overline{1975})$ \\
\hline \multirow[t]{2}{*}{ Sm II } & 7042.206 & 1.076 & -0.760 & $\mathrm{Xu}$ et al. $(2003)$ \\
\hline & 7051.55 & 0.933 & -0.960 & Meggers et al. (1975) \\
\hline \multirow[t]{2}{*}{ Eu II } & 6437.640 & 1.320 & -1.998 & Meggers et al. (1975) \\
\hline & 6645.067 & 1.380 & -1.823 & Meggers et al. (1975) \\
\hline \multirow[t]{2}{*}{ Pr II } & 5219.045 & 0.795 & -0.053 & Meggers et al. (1975) \\
\hline & 5322.772 & 0.483 & -0.141 & Meggers et al. (1975) \\
\hline
\end{tabular}

